\title{
High-Resolution PFI-ZEKE Photoelectron Spectroscopic Study of the Two Lowest Electronic States of the Ozone Cation $\mathrm{O}_{3}^{+}$
}

\author{
S. Willitsch ${ }^{a}$, F. Innocenti ${ }^{b}$, J. M. Dyke ${ }^{b}$ and F. Merkt ${ }^{a, *}$
}

${ }^{a}$ Physical Chemistry

ETH Zurich

8093 Zurich

Switzerland

$b$ Department of Chemistry

The University

Southampton SO17 1BJ

United Kingdom

* Author to whom correspondence should be addressed. 


\begin{abstract}
The pulsed-field-ionization zero-kinetic-energy (PFI-ZEKE) photoelectron spectrum of jet-cooled $\mathrm{O}_{3}$ has been recorded in the range $101000-104000 \mathrm{~cm}^{-1}$. The origins of the $\tilde{\mathrm{X}}{ }^{1} \mathrm{~A}_{1} \rightarrow \tilde{\mathrm{X}}^{+}{ }^{2} \mathrm{~A}_{1}$ and $\tilde{\mathrm{X}}{ }^{1} \mathrm{~A}_{1} \rightarrow \tilde{\mathrm{A}}^{+}{ }^{2} \mathrm{~B}_{2}$ transitions could be determined from the rotational structure of the bands, the photoionization selection rules, the photoionization efficiency curve and comparison with ab initio calculations. The first adiabatic ionization energy of $\mathrm{O}_{3}$ was determined to be $101020.5(5) \mathrm{cm}^{-1}$ and the energy difference between the $\tilde{\mathrm{X}}^{+2} \mathrm{~A}_{1}(0,0,0)$ and $\tilde{\mathrm{A}}^{+}{ }^{2} \mathrm{~B}_{2}(0,0,0)$ states to be $T_{0} / h c=1089.7(4) \mathrm{cm}^{-1}$. Whereas the $\tilde{\mathrm{X}} \rightarrow \tilde{\mathrm{X}}^{+}$transition consists of an intense and regular progression in the bending $\left(\nu_{2}\right)$ mode observed up to $v_{4}^{+}=4$, only the origin of the $\tilde{\mathrm{X}} \rightarrow \tilde{\mathrm{A}}^{+}$transition was observed. The analysis of the rotational structure led to the derivation of the $r_{0}$ structure of $\mathrm{O}_{3}^{+}$in the $\tilde{\mathrm{X}}^{+}\left(C_{2 \mathrm{v}}, r_{0}=1.25(2) \AA, \alpha_{0}=131.5(9)^{\circ}\right)$ and $\tilde{\mathrm{A}}^{+}\left(C_{2 \mathrm{v}}, r_{0}=1.37(5) \AA, \alpha_{0}=111.3(4)^{\circ}\right)$ states. The appearance of the spectrum, which is regular up to $102300 \mathrm{~cm}^{-1}$, changes abruptly at $\approx 102500 \mathrm{~cm}^{-1}$, a position above which the spectral density increases markedly and the rotational structure of the bands collapses. On the basis of ab initio calculations, this behavior is attributed to the onset of large-amplitude motions spreading through several local minima all the way to large internuclear distances. The $a b$ initio calculations are consistent with earlier results in predicting a seam of conical intersections between the $\tilde{\mathrm{X}}^{+}$ and $\tilde{\mathrm{A}}^{+}$states $\approx 2600 \mathrm{~cm}^{-1}$ above the cationic ground state and demonstrate for the first time the existence of potential minima at large internuclear distances that are connected to the main minima of the $\tilde{\mathrm{X}}^{+}$and $\tilde{\mathrm{A}}^{+}$states through low-lying barriers.
\end{abstract}




\section{Introduction}

Ozone $\mathrm{O}_{3}$ plays a fundamental role in the physics and chemistry of the earth's atmosphere [1] and its spectroscopic properties have been studied in great detail (see, e.g., Refs. [2, 3, 4] and references cited therein). In contrast to the neutral molecule, little is known about the ozone cation $\mathrm{O}_{3}^{+}$until now. In atmospheric chemistry, the knowledge of the energy level structure of the cation is of importance for the analysis of the Rydberg spectrum of $\mathrm{O}_{3}$ [5] and the ion chemistry of $\mathrm{O}_{3}^{+}$[6]. From a spectroscopic point of view, $\mathrm{O}_{3}^{+}$is of interest as an isoelectronic species to $\mathrm{NO}_{2}$, which has been studied intensively experimentally as well as theoretically (see, e.g., Refs. [7, 8, 9]). $\mathrm{NO}_{2}$ represents a prototypical molecular system to characterize the spectroscopic consequences of a conical intersection between

two electronic states (in this case the $\tilde{\mathrm{X}}$ and the $\tilde{\mathrm{A}}$ state). In $\mathrm{NO}_{2}$, the crossing point is located in the near infrared $\left(\approx 10000 \mathrm{~cm}^{-1}\right.$ above the ground state) and leads to extensive perturbations of the vibronic structure in the entire visible spectrum [8].

The $\mathrm{O}_{3}^{+}$cation has been studied using conventional photoelectron spectroscopy $[10,11$, $12,13,14,15]$, photoionization mass spectrometry [16, 17], photofragment spectroscopy [18] and electron impact ionization [19]. Already the early HeI photoelectron spectroscopic studies showed that the lowest three cationic electronic states $1{ }^{2} \mathrm{~A}_{2}, 1{ }^{2} \mathrm{~A}_{1}$ and $1{ }^{2} \mathrm{~B}_{2}$ are located within an energy interval of only about $1 \mathrm{eV}$ (the lowest two states are almost degenerate and could not be resolved in the HeI spectra). However, the energetic ordering of these three states has not been unambiguously established yet. By an analysis of the photoelectron angular distributions, Katsumata et al. [14] were able to assign the third 
photoelectron band to the $\tilde{\mathrm{B}}^{+}{ }^{2} \mathrm{~A}_{2}$ state. Although the ordering of the first two states is predicted to be $\tilde{\mathrm{X}}^{+2}{ }^{2} \mathrm{~A}_{1}$ and $\tilde{\mathrm{A}}^{+}{ }^{2} \mathrm{~B}_{2}$ by recent ab initio calculations [20, 21, 22], this prediction remains to be confirmed by experimental data.

The molecular orbitals of ozone relevant for the interpretation of the photoelectron spectrum are depicted in Fig. 1. Early ab initio calculations of its electronic structure already showed that several configurations contribute to the electronic wavefunction of the ground state of $\mathrm{O}_{3}$ [23]. The leading configurations of the CASSCF wavefunction are [24]

$$
\left(1 \mathrm{a}_{1}\right)^{2}\left(1 \mathrm{~b}_{2}\right)^{2}\left(2 \mathrm{a}_{1}\right)^{2}\left(3 \mathrm{a}_{1}\right)^{2}\left(2 \mathrm{~b}_{2}\right)^{2}\left(4 \mathrm{a}_{1}\right)^{2}\left(5 \mathrm{a}_{1}\right)^{2}\left(1 \mathrm{~b}_{1}\right)^{2}\left(3 \mathrm{~b}_{2}\right)^{2}\left(4 \mathrm{~b}_{2}\right)^{2}\left(6 \mathrm{a}_{1}\right)^{2}\left(1 \mathrm{a}_{2}\right)^{2}\left(2 \mathrm{~b}_{1}\right)^{0} \quad(\approx 81 \%)
$$

and

$$
\ldots\left(4 \mathrm{~b}_{2}\right)^{2}\left(6 \mathrm{a}_{1}\right)^{2}\left(1 \mathrm{a}_{2}\right)^{0}\left(2 \mathrm{~b}_{1}\right)^{2} \quad(\approx 10 \%)
$$

In a single-determinantal picture, the lowest three cationic electronic states $1{ }^{2} \mathrm{~A}_{2}, 1^{2} \mathrm{~A}_{1}$ and $1{ }^{2} \mathrm{~B}_{2}$ arise from the ionization out of the $1 \mathrm{a}_{2}, 6 \mathrm{a}_{1}$ and $4 \mathrm{~b}_{2}$ molecular orbitals, respectively. As in the ground state of the neutral molecule, several configurations significantly contribute to the electronic wavefunctions of these cationic states. The CASSCF wavefunction of the $\tilde{\mathrm{X}}^{+2} \mathrm{~A}_{1}$ state consists of the dominant configurations (see Section 4)

$$
\begin{aligned}
& \ldots\left(4 \mathrm{~b}_{2}\right)^{2}\left(6 \mathrm{a}_{1}\right)^{1}\left(1 \mathrm{a}_{2}\right)^{2}\left(2 \mathrm{~b}_{1}\right)^{0} \quad(\approx 72 \%), \\
& \ldots\left(4 \mathrm{~b}_{2}\right)^{2}\left(6 \mathrm{a}_{1}\right)^{1}\left(1 \mathrm{a}_{2}\right)^{0}\left(2 \mathrm{~b}_{1}\right)^{2} \quad(\approx 4 \%), \\
& \ldots\left(4 \mathrm{~b}_{2}\right)^{1}\left(6 \mathrm{a}_{1}\right)^{2}\left(1 \mathrm{a}_{2}\right)^{1}\left(2 \mathrm{~b}_{1}\right)^{1} \quad(\approx 5 \%),
\end{aligned}
$$


and the most important configurations of the $\tilde{\mathrm{A}}^{+2} \mathrm{~B}_{2}$ state wavefunction are

$$
\begin{aligned}
& \ldots\left(4 \mathrm{~b}_{2}\right)^{1}\left(6 \mathrm{a}_{1}\right)^{2}\left(1 \mathrm{a}_{2}\right)^{2}\left(2 \mathrm{~b}_{1}\right)^{0} \quad(\approx 69 \%), \\
& \ldots\left(4 \mathrm{~b}_{2}\right)^{2}\left(6 \mathrm{a}_{1}\right)^{1}\left(1 \mathrm{a}_{2}\right)^{1}\left(2 \mathrm{~b}_{1}\right)^{1} \quad(\approx 7 \%), \\
& \ldots\left(4 \mathrm{~b}_{2}\right)^{1}\left(6 \mathrm{a}_{1}\right)^{2}\left(1 \mathrm{a}_{2}\right)^{0}\left(2 \mathrm{~b}_{1}\right)^{2} \quad(\approx 4 \%) .
\end{aligned}
$$

Because of configuration interaction, the ordering of the $\tilde{\mathrm{X}}^{+2} \mathrm{~A}_{1}, \tilde{\mathrm{A}}^{+2} \mathrm{~B}_{2}$ and $\tilde{\mathrm{B}}^{+2} \mathrm{~A}_{2}$ states predicted theoretically does not correspond to the energetic ordering of the $4 \mathrm{~b}_{2}, 6 \mathrm{a}_{1}$ and $1 \mathrm{a}_{2}$ molecular orbitals of the neutral molecule.

The previous HeI photoelectron spectroscopic studies did not allow a detailed characterization of the vibronic structure of the $\tilde{\mathrm{X}}^{+}$and $\tilde{\mathrm{A}}^{+}$states. At the resolution of about $200 \mathrm{~cm}^{-1}$ achieved in these studies, the first photoelectron band consists of partially resolved features which were assigned to progressions in the bending modes of both states. The irregular spacings between the bands were either explained by Renner-Teller coupling [12] or by the large-amplitude motion in a double minimum potential [14]. The vibronic structure observed in these spectra was analyzed by Köppel, Cederbaum and co-workers using a vibronic coupling model and analytical potentials for the $\tilde{\mathrm{X}}^{+}$and $\tilde{\mathrm{A}}^{+}$states derived from ab initio calculations $[20,25,26,27]$. In these studies, the irregular structure of the first photoelectron band was attributed to non-adiabatic effects in the vicinity of a conical intersection between the $\tilde{\mathrm{X}}^{+}$and $\tilde{\mathrm{A}}^{+}$states which was calculated to lie $\approx 2500 \mathrm{~cm}^{-1}$ above the minimum of the $\tilde{\mathrm{X}}^{+}$state potential.

The adiabatic ionization energy of $\mathrm{O}_{3}$ has also not been unambiguously determined up 
to now. In their HeI photoelectron spectra, Dyke et al. [12] and Katsumata et al. [14] observed a weak band at $12.44 \mathrm{eV}$ which was assigned to the transition to the vibronic ground state of the cation. This weak band was not observed in other studies $[11,13]$ in which a value of $12.52 \mathrm{eV}$ was reported in agreement with the result of $12.519 \pm 0.004 \mathrm{eV}$ obtained by photoionization mass spectrometry [16]. A value near $12.52 \mathrm{eV}$ has also been suggested on the basis of an analysis of the relevant thermochemical cycles $[18,19]$. The simulation of the intensity distribution of the first photoelectron band led Müller et al. to propose that the spectral feature near $12.44 \mathrm{eV}$ actually corresponds to a hot band [27].

In the present study, the pulsed-field-ionization zero-kinetic-energy (PFI-ZEKE) photoelectron spectrum of $\mathrm{O}_{3}$ was recorded with a resolution two to three orders of magnitude higher than in previous investigations. The high resolution enabled the observation of the rotational structure of many bands. The analysis of this structure turned out to be the key to the assignment of the vibronic symmetries of the cationic states using photoionization selection rules and allowed the unambiguous determination of the energetic ordering of the ${ }^{2} \mathrm{~A}_{1}$ and ${ }^{2} \mathrm{~B}_{2}$ states, of their corresponding adiabatic ionization energies and of the lowest dissociation energy of the cation. The potential energy surface of the lowest state was explored by theoretical methods to gain insight into the mechanisms that are responsible for the complex and dense vibronic structure that is observed at excitation energies $>1500$ $\mathrm{cm}^{-1}$ 


\section{Experimental}

A detailed description of the extreme-ultraviolet (XUV) PFI-ZEKE photoelectron spectrometer has been given previously [28] and only aspects relevant to the present study are discussed here. Tunable XUV radiation in the range $101000-104000 \mathrm{~cm}^{-1}$ was generated by resonance-enhanced sum-frequency mixing in a xenon gas beam using the $(5 \mathrm{p})^{5}\left({ }^{2} \mathrm{P}_{3 / 2}\right) 6 \mathrm{p}[1 / 2](J=0) \leftarrow(5 \mathrm{p})^{6}{ }^{1} \mathrm{~S}_{0}$ two-photon resonance at $2 \tilde{\nu}_{1}=80118.974 \mathrm{~cm}^{-1}$.

Ozone was generated by a discharge of oxygen (purity 99.999\%, Pangas) in a homemade ozonizer (Southampton) and stored on silica gel in a U-tube at $-79{ }^{\circ} \mathrm{C}$. To transfer the substance into the measurement chamber, the U-tube was attached to the gas inlet of the spectrometer using glass and teflon-coated stainless steel tubing. The ozone was desorbed from the silica gel at $-15{ }^{\circ} \mathrm{C}$, seeded into a stream of helium at a pressure of 1.3 bar and introduced into the photoionization region by means of a skimmed pulsed supersonic expansion. The rotational temperature of $\mathrm{O}_{3}$ was determined to be $T_{\text {rot }}=6 \mathrm{~K}$ from the rotational structure of the photoelectron spectra. A partial decomposition of ozone in the tubes could not be avoided leading to a small amount of oxygen in the gas sample. The gas mixture was renewed before each scan.

PFI-ZEKE photoelectron spectra were recorded following single-photon XUV excitation from rotational levels of its vibronic ground state to very high Rydberg states located below the successive ionization thresholds by monitoring the wavenumber-dependent electron signal produced by pulsed-field ionization [29] using a two-pulse electric field sequence. The first pulse with an amplitude of $+100 \mathrm{mV} / \mathrm{cm}$ and a duration of $500 \mathrm{~ns}$ was triggered 
500 ns after the XUV laser pulse and served the purposes of removing spurious free electrons from the photoionization region and of narrowing down the range of Rydberg states which are field ionized by the subsequent pulse [30]. The second field pulse with an amplitude of $-200 \mathrm{mV} / \mathrm{cm}$ was applied immediately after the first and the electrons released by this pulse were monitored by setting a suitable detection gate in the electron time-of-flight spectrum. This pulse sequence enabled a resolution of $\Gamma_{\mathrm{FWHM}}=0.6 \mathrm{~cm}^{-1}$ in the PFI-ZEKE photoelectron spectra. Selected bands were recorded at a higher resolution of $\Gamma_{\mathrm{FWHM}}=0.4$ $\mathrm{cm}^{-1}$ by decreasing the amplitude of the second pulse to $-120 \mathrm{mV} / \mathrm{cm}$.

Apart from the spectral features of ozone, the PFI-ZEKE photoelectron spectrum also contains bands of water and oxygen which appear as impurities in the gas sample. $\mathrm{O}_{2}$ stems from the decomposition of ozone in the gas system and $\mathrm{H}_{2} \mathrm{O}$ from moisture trapped on the silica gel. Baking the U-tube at $140^{\circ}$ before each loading with ozone did not eliminate the contamination with $\mathrm{H}_{2} \mathrm{O}$. To distinguish the bands of ozone from those of these impurities, mass-analyzed threshold-ionization (MATI) spectra of selected bands were recorded by monitoring the wavenumber dependent $\mathrm{O}_{3}^{+}$ion signal generated by pulsed-field ionization of high Rydberg states [31] with a two-pulse electric field sequence. The first pulse with an amplitude of $-780 \mathrm{mV} / \mathrm{cm}$ and a duration of $3.0 \mu$ s was triggered $100 \mathrm{~ns}$ after the VUV excitation and served the purpose of separating prompt $\mathrm{O}_{3}^{+}$ions from the Rydberg molecules. The second pulse with an amplitude of $+43.1 \mathrm{~V} / \mathrm{cm}$ was used to ionize the Rydberg species and extract the ions. The MATI spectra were not as highly resolved as the PFI-ZEKE photoelectron spectra and primarily served the purpose of identifying the bands of ozone. 
The absolute wavenumber calibration was achieved by the simultaneous recording of optogalvanic spectra of neon and making reference to the line positions tabulated in Ref. $[32]$.

\section{PFI-ZEKE photoelectron spectra and their inter-}

\section{pretation}

\subsection{Vibronic Structure}

A survey PFI-ZEKE photoelectron spectrum of the lowest two electronic states of the ozone cation is shown in Fig. 2. The spectrum also contains bands of the impurities $\mathrm{O}_{2}$ and $\mathrm{H}_{2} \mathrm{O}$, which could easily be identified by comparison with their known spectra [33, 34]. These bands were not present in the MATI spectrum which is compared with the PFIZEKE photoelectron spectrum in Fig. 3. All other spectral structures of the PFI-ZEKE photoelectron spectrum could be attributed to ozone on the basis of the MATI spectrum and of the analysis of the rotational structures of the bands. The intensity of the XUV radiation was too low for spectra of sufficient quality to be recorded in the region from $103000-103250 \mathrm{~cm}^{-1}[35]$. The intensity scale of the spectrum beyond $103250 \mathrm{~cm}^{-1}$ could only be approximately matched to that of the region below $103000 \mathrm{~cm}^{-1}$.

Because of the uncertainty in the literature concerning the precise value of the first adiabatic ionization energy of $\mathrm{O}_{3}$, special care was taken in the identification of the origin band of the PFI-ZEKE photoelectron spectrum. In Fig. 4, a detailed view of the region of 
the lowest band observed in the PFI-ZEKE photoelectron spectrum of Fig. 2 is compared on a larger scaler with the photoionization (PI) spectrum recorded by monitoring the $\mathrm{O}_{3}^{+}$photoion signal as a function of the XUV wavenumber. The PI spectrum shows a clear step rising from a signal level of zero over the first PFI-ZEKE photoelectron band which indicates that this band corresponds to the first adiabatic component. The signalto-noise ratio of our PI measurement would have been sufficiently high to observe a step in the PI spectrum corresponding to the first weak band observed at $12.44 \mathrm{eV}$ by Dyke et al. [12] and Katsumata et al. [14]. We attribute this apparent contradiction to the different temperatures of the $\mathrm{O}_{3}$ sample and conclude that the first band observed in the HeI photoelectron spectra of room temperature $\mathrm{O}_{3}$ represents a hot band, as already suggested by Müller et al. [27].

Three types of bands are observed in the PFI-ZEKE photoelectron spectrum:

First, in the low wavenumber region of the spectrum (see upper panel of Fig. 2), a regular progression of intense bands is observed which exhibit almost identical rotational structures and thus correspond to cationic states of the same vibronic symmetry. The average spacing of about $620 \mathrm{~cm}^{-1}$ between the bands is similar to the fundamental bending wavenumber $\tilde{\nu}_{2}=700.931 \mathrm{~cm}^{-1}$ of neutral $\mathrm{O}_{3}[3]$ and corresponds well to the harmonic bending wavenumber of $634 \mathrm{~cm}^{-1}$ predicted in $\operatorname{CASSCF}(17,12) /$ aug-cc-pVTZ ab initio calculations (see Section 4). Hence, these bands can be confidently assigned to a progression in the bending mode $\left(0, v_{2}^{+}, 0\right)$ of the $\tilde{\mathrm{X}}^{+}$state of the cation. The progression is observed up to the $(0,4,0)$ band at $102484 \mathrm{~cm}^{-1}$.

Second, a weaker feature at $102110 \mathrm{~cm}^{-1}$ is observed which possesses a different rota- 
tional structure and hence must be attributed to a cationic state of a different vibronic symmetry than the bands of the bending progression in the $\tilde{\mathrm{X}}^{+}$state. This band was assigned to the origin band of the $\tilde{\mathrm{A}}^{+}$state $\left(\tilde{\mathrm{A}}^{+}(0,0,0)\right)$ on the basis of an analysis of the rotational structure as will be discussed in detail in Section 3.2.2. No other band showing a similar rotational structure could be identified in the entire spectrum.

Third, the spectrum in the region $>102600 \mathrm{~cm}^{-1}$ is dominated by a multitude of narrow features. Their rotational structures are all similar but are very different from those of the $\tilde{\mathrm{X}}^{+}\left(0, v_{2}^{+}, 0\right)$ and $\tilde{\mathrm{A}}^{+}(0,0,0)$ bands. As will be discussed in Section 4 , the vibronic structure in this region of the spectrum is likely to be strongly influenced by the non adiabatic coupling between the $\tilde{\mathrm{X}}^{+}$and $\tilde{\mathrm{A}}^{+}$states and by large amplitude motions. Because of the high density of vibronic states and the very irregular appearance of the spectrum, no conclusive assignment of these bands could be reached yet.

\subsection{Rotational Structure}

\subsubsection{The $\tilde{\mathrm{X}}^{+2} \mathrm{~A}_{1}\left(0, v_{2}^{+}, 0\right)$ bands}

A detailed view of the first band in the PFI-ZEKE photoelectron spectrum is shown in Fig. 5. The well resolved rotational structure has been assigned on the basis of an analysis of lower state combination differences and of simulations of the rotational structure after successive refinements of the ionic rotational constants.

In Table 1, the rotational selection rules for photoionizing transitions from the vibronic ground state of neutral $\mathrm{O}_{3}$ to vibronic states of the cation with symmetry $\Gamma_{\mathrm{ve}}^{+}$are given 
in terms of the changes of the asymmetric top quantum numbers $\Delta K_{a}=K_{a}^{+}-K_{a}^{\prime \prime}$ and $\Delta K_{c}=K_{c}^{+}-K_{c}^{\prime \prime}$. The selection rules have been derived from the general rovibronic photoionization selection rules [36] in the molecular symmetry (MS) groups $C_{2 \mathrm{v}}(\mathrm{M})$ and $C_{\mathrm{s}}(\mathrm{M})$. The linear configuration (with its coresponding symmetry group $D_{\infty \mathrm{h}}(\mathrm{M})$ ) is not accessible in the cation at the excitation energies of less than $4000 \mathrm{~cm}^{-1}$ investigated in the present study [20] and is hence not considered further. Because the electronic symmetries $\Gamma_{\mathrm{e}}^{+}$of the first two cationic states are $\mathrm{A}_{1}$ and $\mathrm{B}_{2}$ in $C_{2 \mathrm{v}}(\mathrm{M})$ and the symmetries of the normal modes are also either $\mathrm{a}_{1}$ (symmetric stretch $\nu_{1}$ and bend $\nu_{2}$ ) or $\mathrm{b}_{2}$ (asymmetric stretch $\nu_{3}$ ), only vibronic states with symmetries $\Gamma_{\mathrm{ve}}^{+}=\mathrm{A}_{1}$ and $\mathrm{B}_{2}$ need to be considered. Both of these irreducible representations correlate with $\mathrm{A}^{\prime}$ in $C_{\mathrm{s}}(\mathrm{M})$. From Table 1, it can be seen that the rotational photoionization selection rules vary according to the vibronic symmetry of the cationic state and the MS group in which they are derived. Thus, by an analysis of the rotational structure it is possible to determine the vibronic symmetry of the cationic state and the relevant MS group.

The assignment bars in Fig. 5 denote the most intense transitions using the notation $N_{K_{a}^{\prime \prime} K_{c}^{\prime \prime}}^{\prime \prime} \rightarrow N_{K_{a}^{+} K_{C}^{+}}^{+}$, where $N$ represents the quantum number of the total angular momentum without spin and " and + denote the neutral and ionic states, respectively. Only transitions with $\Delta K_{a} \Delta K_{c}=$ ee and oo have been observed, which indicates that the vibronic symmetry of the cationic state is $\Gamma_{\mathrm{ve}}^{+}=\mathrm{A}_{1}$ and that the symmetry group is $C_{2 \mathrm{v}}(\mathrm{M})$ (see Table 1). Because the symmetry of the vibrational ground state is always $A_{1}$, it is straightforward to determine the electronic symmetry of the cationic state to be $\mathrm{A}_{1}$. Thus, the analysis of the rotational structure of the high-resolution photoelectron spectrum en- 
abled for the first time the assignment of the symmetry of the lowest electronic state of $\mathrm{O}_{3}^{+}$based solely on experimental data.

The rotational structure was analyzed in terms of a rigid asymmetric rotor Hamiltonian for the neutral and the cationic states:

$$
\mathbf{H}_{\mathrm{rot}}=A \mathbf{N}_{a}^{2}+B \mathbf{N}_{b}^{2}+C \mathbf{N}_{c}^{2}
$$

The rotational constants $A^{+}, B^{+}$and $C^{+}$of the cationic state and the adiabatic ionization energy (corresponding to the $0_{00} \rightarrow 0_{00}$ transition) were fitted to the experimental line positions at fixed values of the neutral state constants $A^{\prime \prime}, B^{\prime \prime}$ and $C^{\prime \prime}[2]$. The results of the fit are listed in Table 2. The values of the fitted $B^{+}$and $C^{+}$constants were identical within the statistical uncertainty so that only the mean value $\left(B^{+}+C^{+}\right) / 2$ is given. The adiabatic ionization energy was determined to be $I E / h c=101020.5(5) \mathrm{cm}^{-1}$. Assuming $C_{2 \mathrm{v}}$ symmetry, an experimental $r_{0}$ geometry was derived from the rotational constants (bond length $r_{0}=1.250(20) \AA$, bond angle $\left.\alpha_{0}=131.49(91)^{\circ}\right)$.

The lower trace of Fig. 5 represents a simulation of the rotational structure using the fitted rotational constants and a recently developed model for the rovibronic photoionization cross sections $\sigma$ of asymmetric top molecules [37]:

$$
\begin{array}{r}
\sigma \propto \rho^{\prime \prime} q_{\mathrm{v}}^{2} \sum_{\left|\lambda^{\prime \prime}\right| \leq \ell^{\prime \prime}}^{\infty} \frac{2 N^{+}+1}{2 \ell^{\prime \prime}+1}\left[\sum_{K^{+}, K^{\prime \prime}}(-1)^{K^{+}} c_{K^{+}}^{N^{+}, K_{a}^{+}, K_{c}^{+}} c_{K^{\prime \prime}}^{N^{\prime \prime}, K_{a}^{\prime \prime}, K_{c}^{\prime \prime}}\left(\begin{array}{ccc}
N^{+} & \ell^{\prime \prime} & N^{\prime \prime} \\
-K^{+} & \lambda^{\prime \prime} & K^{\prime \prime}
\end{array}\right)\right]^{2} \\
\times\left|C_{\ell^{\prime \prime}, \lambda^{\prime \prime}}^{\left(\alpha^{\prime \prime}\right)}\right|^{2}\left[\ell^{\prime \prime}\left|F_{\alpha^{\prime \prime} \ell^{\prime \prime}}^{E, \ell^{\prime \prime}-1}\right|^{2}+\left(\ell^{\prime \prime}+1\right)\left|F_{\alpha^{\prime \prime} \ell^{\prime \prime}}^{E, \ell^{\prime \prime}+1}\right|^{2}\right] .
\end{array}
$$


Eq. (6) relies on the assumption that the photoelectron is ejected out of a definite molecular orbital $\phi_{\alpha^{\prime \prime}}$ which is expressed as a single-center expansion at the molecular center of mass with the expansion coefficients $C_{\ell^{\prime \prime}, \lambda^{\prime \prime}}^{\left(\alpha^{\prime \prime}\right)} . \ell^{\prime \prime}$ and $\lambda^{\prime \prime}$ are the quantum numbers of the orbital angular momenta of the components of the single-center expansion and their projection on the molecular axis. $q_{\mathrm{v}}^{2}$ stands for the vibrational part of the transition moment and $c_{K}^{N, K_{a}, K_{c}}$ represent the expansion coefficients of the asymmetric top wavefunction in a symmetric top basis $|N, K\rangle$, where $K$ denotes the quantum number of the moleculefixed projection of $\overrightarrow{\mathbf{N}}$. $\rho^{\prime \prime}$ is the population of the lower state which is expressed as a product of the nuclear spin statistical weight and a Boltzmann factor. The $F_{\alpha^{\prime \prime} \ell^{\prime \prime}}^{E, \ell^{\prime \prime} \pm 1}$ represent radial transition integrals as defined in Eq. (25) of Ref. [38]. Given that for the present purpose only relative rotational line intensities are of interest, the electronic factors $B_{\ell^{\prime \prime}, \lambda^{\prime \prime}}^{\left(\alpha^{\prime \prime}\right)}=\left|C_{\ell^{\prime \prime}, \lambda^{\prime \prime}}^{\left(\alpha^{\prime \prime}\right)}\right|^{2}\left[\ell^{\prime \prime}\left|F_{\alpha^{\prime \prime} \ell^{\prime \prime}}^{E, \ell^{\prime \prime}}-1\right|^{2}+\left(\ell^{\prime \prime}+1\right)\left|F_{\alpha^{\prime \prime} \ell^{\prime \prime}}^{E, \ell^{\prime \prime}+1}\right|^{2}\right]$ are treated as effective parameters and are adjusted to reproduce the experimental spectrum. Angular momentum contributions of other configurations in the CI wavefunction are taken into account indirectly if they make a contribution to the $B_{\ell^{\prime \prime}, \lambda^{\prime \prime}}^{\left(\alpha^{\prime \prime}\right)}$ coefficients.

In the simulation of the $\tilde{\mathrm{X}}^{+}(0,0,0)$ band, it was assumed that photoionization occurs out of the $6 \mathrm{a}_{1}$ molecular orbital, because the CI expansion of the electronic wavefunction of the cationic state is dominated by the ...( $\left(4 \mathrm{~b}_{2}\right)^{2}\left(6 \mathrm{a}_{1}\right)^{1}\left(1 \mathrm{a}_{2}\right)^{2}\left(2 \mathrm{~b}_{1}\right)^{0}$ configuration (see Section 1 ). The $6 \mathrm{a}_{1}$ orbital exhibits a strongly mixed angular momentum composition (see Fig. 1) and the single-center expansion converges slowly. The best agreement with the experimental spectrum could be achieved by taking into account a total of seven angular momentum components $\left(\ell^{\prime \prime}, \lambda^{\prime \prime}\right):(0,0)$ (with the coefficient $\left.\left|B_{0,0}^{\left(6 a_{1}\right)}\right|=0.16\right),(1,0)\left(\left|B_{1,0}^{\left(6 a_{1}\right)}\right|=0.29\right)$, 
$(1, \pm 1)\left(\left|B_{1, \pm 1}^{\left(6 a_{1}\right)}\right|=0.29\right),(2,0)\left(\left|B_{2,0}^{\left(6 a_{1}\right)}\right|=0.29\right),(2, \pm 1)\left(\left|B_{2, \pm 1}^{\left(6 a_{1}\right)}\right|=0.29\right),(2, \pm 2)\left(\left|B_{2, \pm 2}^{\left(6 a_{1}\right)}\right|=\right.$

$0.24)$ and $(3, \pm 1)\left(\left|B_{3, \pm 1}^{\left(6 a_{1}\right)}\right|=0.29\right)$. Because of the large number of terms in the singlecenter expansion and the spectral congestion of the experimental spectrum, the coefficients $B_{\ell^{\prime \prime}, \lambda^{\prime \prime}}^{\left(\alpha^{\prime \prime}\right)}$ could only be determined with an accuracy of about $20 \%$.

The good agreement between the simulated and experimental spectra confirms the assumption of the mixed angular momentum composition of the $6 \mathrm{a}_{1}$ molecular orbital and indicates that the photoelectron is ejected as a superposition of even and odd partial wave components with significant contributions up to $\ell=4$.

The results of the analysis of the rotational structures of the bands associated with transitions to excited bending levels $\left(0, v_{2}^{+}, 0\right)$ of the $\tilde{\mathrm{X}}^{+}$state are listed in Table 3 . The $A^{+}$ rotational constants of the $(0,3,0)$ and $(0,4,0)$ bands are considerably smaller than those of the $(0,0,0),(0,1,0)$ and $(0,2,0)$ bands, which suggests that the rotational structures of these higher excited bending levels are probably perturbed. Moreover, the wavenumber difference between the $(0,4,0)$ and $(0,3,0)$ bands is larger than the fundamental bending wavenumber (see Table 3). Possible causes for these perturbations are discussed in Section 4.

\subsubsection{The $\tilde{\mathrm{A}}^{+}{ }^{2} \mathrm{~B}_{2}(\mathbf{0 , 0 , 0 )}$ band}

The PFI-ZEKE photoelectron spectrum of the $\tilde{\mathrm{X}}^{1} \mathrm{~A}_{1}(0,0,0) \rightarrow \tilde{\mathrm{A}}^{+}{ }^{2} \mathrm{~B}_{2}(0,0,0)$ transition at $102110 \mathrm{~cm}^{-1}$ is displayed in Fig. 6. The rotational structure of this band differs strongly from that of the bending progression in the $\tilde{\mathrm{X}}^{+}$state, which indicates a different vibronic symmetry of the cationic state. The assignment bars in Fig. 6 denote the most intense 
transitions. Only transitions with $\Delta K_{a} \Delta K_{c}=$ eo and oe are observed. Hence, it can be concluded that the symmetry group is $C_{2 \mathrm{v}}(\mathrm{M})$ and that the vibronic symmetry of the upper state is $\mathrm{B}_{2}$ (see Table 1 ). The rotational constants and the adiabatic ionization energy were determined by a fit of Eq. (5) to the observed line positions and are listed in Table 2. Because of the higher spectral congestion, the statistical uncertainties of the fitted parameters are larger than for the $\tilde{\mathrm{X}}^{+}(0,0,0)$ band. The adiabatic ionization energy was determined to be $I E / h c=102110.1(6) \mathrm{cm}^{-1}$. The largest rotational constant $A^{+}=$ $2.65(26) \mathrm{cm}^{-1}$ is considerably smaller than for the $\tilde{\mathrm{X}}^{+}(0,0,0)$ state $\left(A^{+}=5.99(10) \mathrm{cm}^{-1}\right)$ and the $r_{0}$ geometry derived from the rotational constants is $r_{0}=1.370(52) \AA$ and $\alpha_{0}=111.3(38)^{\circ}$.

The lower trace in Fig. 6 represents a simulation of the rotational structure using the fitted rotational constants and Eq. (6). It was assumed that the electron is removed from the $4 b_{2}$ molecular orbital of the neutral molecule (the electronic wavefunction of the $\tilde{\mathrm{A}}^{+}$state is dominated by the ...( $\left(4 \mathrm{~b}_{2}\right)^{1}\left(6 \mathrm{a}_{1}\right)^{2}\left(1 \mathrm{a}_{2}\right)^{2}\left(2 \mathrm{~b}_{1}\right)^{0}$ configuration, see Section 1$)$. The best agreement with the experimental spectrum could be achieved by assuming that two angular momentum components $\left(\ell^{\prime \prime}, \lambda^{\prime \prime}\right)=(1,0)$ and $(2, \pm 1)$ contribute to the singlecenter expansion of the $4 \mathrm{~b}_{2}$ orbital with equal weights. This implies that the photoelectron is mainly ejected as a superposition of s,d,p and f partial waves upon ionization to the $\tilde{\mathrm{A}}^{+}$ state.

From the adiabatic ionization energies, the energy difference between the $\tilde{\mathrm{X}}^{+}{ }^{2} \mathrm{~A}_{1}$ and $\tilde{\mathrm{A}}^{+2} \mathrm{~B}_{2}$ states can be determined to be $\Delta T_{0} / h c=1089.5(4) \mathrm{cm}^{-1}$. This result is in good agreement with the theoretical value of $\Delta T_{\mathrm{e}} / h c=1037.5 \mathrm{~cm}^{-1}$ (see Section 4).

In summary, the assignment of the band around $102110 \mathrm{~cm}^{-1}$ to the origin band of the 
$\tilde{\mathrm{A}}^{+}$state is based on the following five observations:

1. The vibronic symmetry of the cationic state is $\Gamma_{\mathrm{ve}}^{+}=\mathrm{B}_{2}$.

2. The band is the first with a rotational structure appreciably different from that of the $\tilde{\mathrm{X}}^{+}(0,0,0)$ band. The $\tilde{\mathrm{X}}^{+}(0,0,1)$ state also has a vibronic symmetry $\Gamma_{\mathrm{ve}}^{+}=\mathrm{B}_{2}$; however, the theoretical harmonic wavenumber of $\tilde{\nu}_{3}=838 \mathrm{~cm}^{-1}$ (see Section 4) differs too strongly from the observed wavenumber. Moreover, in first approximation the $\tilde{\mathrm{X}}(0,0,0) \rightarrow \tilde{\mathrm{X}}^{+}(0,0,1)$ band is forbidden because of the vanishing Franck-Condon factor.

3. The observed photoionization propensity rules are compatible with photoionization out of the $4 b_{2}$ molecular orbital of $\mathrm{O}_{3}$.

4. The rotational constants, and therefore the experimental $r_{0}$ geometry, agree with the predictions of $a b$ initio calculations of the $\tilde{\mathrm{A}}^{+}$state, but differ markedly from the rotational constants and $r_{\mathrm{e}}$ structure of the $\tilde{\mathrm{X}}^{+}$state (see Sections 3.2.1 and 4).

5. The experimental and theoretical energy difference between the $\tilde{\mathrm{X}}^{+}$and $\tilde{\mathrm{A}}^{+}$states are in good agreement (see Section 4).

\subsection{Excited vibronic states}

The PFI-ZEKE photoelectron spectrum of two of the higher excited vibronic states is displayed in Fig. 7. The bands exhibit a distinct shape consisting of two sharp and intense lines surrounded by two weaker satellites (see the assignment bars in Fig. 7) which is 
characteristic for most bands observed beyond $>102500 \mathrm{~cm}^{-1}$, the only exceptions being the $\tilde{\mathrm{X}}^{+}(0,3,0)$ and $(0,4,0)$ bands at $102834 \mathrm{~cm}^{-1}$ and $103484 \mathrm{~cm}^{-1}$, respectively. The width of the partially resolved rotational structure of these bands is significantly smaller than for the $\tilde{\mathrm{X}}^{+}\left(0, v_{2}^{+}, 0\right)$ and $\tilde{\mathrm{A}}^{+}(0,0,0)$ bands, which indicates that the effective rotational constants of the upper states are considerably reduced. The strong spectral congestion prevented an unambiguous assignment and the determination of the rotational constants $A^{+}, B^{+}$and $C^{+}$and the ionization energy $I E$ in a fitting procedure. The rotational contour is compatible with values $A^{+}<1.0 \mathrm{~cm}^{-1}$ and $\left(B^{+}+C^{+}\right) / 2<0.3 \mathrm{~cm}^{-1}$. This type of bands is not observed in the wavenumber region $<102600 \mathrm{~cm}^{-1}$ suggesting that a large and abrupt change in the effective molecular geometry takes place at excitation energies of more than $1500 \mathrm{~cm}^{-1}$ with respect to the vibronic ground state. A possible explanation of this behavior will be given in Section 4 .

\subsection{Thermochemical implications}

With the new accurate value for the first adiabatic ionization energy of $\mathrm{O}_{3}$, a more precise estimate for the lowest $\mathrm{O}_{3}^{+} \rightarrow \mathrm{O}_{2}^{+}+\mathrm{O}$ dissociation threshold can be calculated using a thermochemical cycle

$$
\begin{aligned}
D_{0}\left(\mathrm{O}_{2}^{+}-\mathrm{O}\right) / h c & =\left[E_{\mathrm{A}, 0 \mathrm{~K}}\left(\mathrm{O}_{2}^{+}, \mathrm{O}_{3}\right)-I E\left(\mathrm{O}_{3}\right)\right] / h c \\
& =4840 \pm 32 \mathrm{~cm}^{-1}(\hat{=} 0.600 \pm 0.004 \mathrm{eV})
\end{aligned}
$$


and the value of the appearance energy of $\mathrm{O}_{2}^{+}$in the photoionization of $\mathrm{O}_{3}^{+}\left(E_{\mathrm{A}, 0 \mathrm{~K}}\left(\mathrm{O}_{2}^{+}, \mathrm{O}_{3}\right) / h c=\right.$ $\left.105860 \pm 32 \mathrm{~cm}^{-1}[16]\right)$. Alternatively, $D_{0}\left(\mathrm{O}_{2}^{+}-\mathrm{O}\right)$ can be calculated using the adiabatic ionization energy of $\mathrm{O}_{2}\left(I E / h c=97352.2 \pm 1.2 \mathrm{~cm}^{-1}\right)[33]$ and the dissociation energy of $\mathrm{O}_{3}\left(D_{0}\left(\mathrm{O}_{2}-\mathrm{O}\right) / h c=8566 \pm 3 \mathrm{~cm}^{-1}\right)[39]:$

$$
\begin{aligned}
D_{0}\left(\mathrm{O}_{2}^{+}-\mathrm{O}\right) / h c & =\left[D_{0}\left(\mathrm{O}_{2}-\mathrm{O}\right)+\operatorname{IE}\left(\mathrm{O}_{2}\right)-I E\left(\mathrm{O}_{3}\right)\right] / h c \\
& =4898 \pm 3 \mathrm{~cm}^{-1}(\hat{=} 0.6073 \pm 0.0004) \mathrm{eV} .
\end{aligned}
$$

These only slightly different results are in agreement with the previous estimate of $D_{0}\left(\mathrm{O}_{2}^{+}-\mathrm{O}\right)=0.59 \mathrm{eV}$ given by Moseley et al. [18]. This low value for the dissociation energy implies that $\mathrm{O}_{3}^{+}$is a very weakly bound molecule and that the ground state potential energy surface is flat and anharmonic.

\section{Theoretical results}

\subsection{Purpose of the ab initio calculations and computational meth- ods}

The purpose of the ab initio calculations of the potential energy surface of $\mathrm{O}_{3}^{+}$was threefold. First, it enabled a rationalization of the observed rovibronic propensity/selection rules, primarily by providing information on the molecular orbital structure of the neutral and the cation (see Sections 1 and 3). Second, it allowed the independent determination of 
the stationary points on the potential energy surfaces corresponding to the $\tilde{\mathrm{X}}^{+}$and $\tilde{\mathrm{A}}^{+}$ states as well as their rotational constants and vibrational structure. This information corroborated the experimental assignments (see Section 3.2.2). Finally, the exploration of the topographic properties of the potential energy surfaces was used to establish a framework to discuss the as yet unassigned spectral features observed at excitation energies of more than $1500 \mathrm{~cm}^{-1}$ above the vibronic ground state of the cation.

The difficulties associated with accurate electronic structure calculations of neutral $\mathrm{O}_{3}$ have been thoroughly discussed in the literature (see, e.g., Refs. [40, 41] and references cited therein). The high degree of configurational mixing in the electronic wavefunction necessitates a high-level treatment of static and dynamic electron correlation effects, preferably using multireference methods. The situation is analogous for the cation $[21,20]$. Three types of calculations have been carried out in the present investigation:

- Harmonic frequencies were calculated after geometry optimization using the complete active space self consistent field (CASSCF) method [42, 43] and the aug-cc-pVTZ basis set [44]. The active space encompassed the entire valence space (17 electrons in 12 orbitals, $\operatorname{CASSCF}(17,12))$.

- The geometry and energy of the stationary points on the potential energy surfaces were calculated using the multireference configuration interaction method with singles and doubles excitations (MRCI-SD) [45, 46] following CASSCF $(17,12)$ calculations using the aug-cc-pVTZ basis set. In the MRCI procedure, only configuration state functions with coefficients $>0.01$ were retained. 
- Larger regions of the adiabatic potential energy surfaces of the lowest two electronic states were explored by performing single-point calculations on an equidistant grid of different nuclear configurations at the CASSCF and the multireference second order perturbation theory (CASPT2) levels of theory [47]. The lowest two A'surfaces were calculated in $C_{\mathrm{s}}$ geometry in a state-averaged procedure with equal weights. Because these calculations also covered nuclear configurations involving large internuclear distances, a larger basis set (cc-pVQZ) was employed to reduce basis set superposition errors. To compensate for the increased computational cost, the $2 \mathrm{~s}$ orbitals of the oxygen atoms were omitted from the active space in the CASPT2 calculations (CASSPT2(11,9)).

All calculations were performed using the MOLPRO 2002 program package [48].

\subsection{Stationary points}

The results for the calculations of the stationary points of the $\tilde{\mathrm{X}}^{+}{ }^{2} \mathrm{~A}_{1}$ and $\tilde{\mathrm{A}}^{+}{ }^{2} \mathrm{~B}_{2}$ states are collected in Table 4 where they are compared with the present experimental results and the previous theoretical results of Schmelz et al. [20]. Although the zero-point vibrational motion has not been accounted for in the calculations, the theoretical and experimental values for the geometries and the energy difference between the $\tilde{\mathrm{X}}^{+}$and $\tilde{\mathrm{A}}^{+}$states compare favorably, especially for the results using the MRCI method, which represents the most sophisticated method employed in the present study. As already discussed in Section 3.2.2, the good agreement between the calculated and experimental values supports the vibronic 
assignments of the PFI-ZEKE photoelectron spectrum, particularly with regard to the $\tilde{\mathrm{A}}^{+}(0,0,0)$ band. The differences between the calculated and experimental geometries are somewhat larger for the $\tilde{\mathrm{A}}^{+}$state than for the $\tilde{\mathrm{X}}^{+}$state. We attribute this discrepancy to the pronounced anharmonicity of the potential energy surface in the vicinity of the $\tilde{\mathrm{A}}^{+}$ state minimum (see Section 4.3).

\subsection{Topography of the lowest two potential surfaces}

As an extension to the CASSCF calculations of the lowest two potential energy surfaces of $\mathrm{O}_{3}^{+}$performed by Schmelz et al. [20] in $C_{2 \mathrm{v}}$ geometry, our calculations also covered the $C_{\mathrm{s}}$ space where the $\mathrm{O}-\mathrm{O}$ bond lengths $r_{1}$ and $r_{2}$ are unequal $\left(r_{1} \neq r_{2}\right)$.

In Fig. 8, the bending potential curves of the $\tilde{\mathrm{X}}^{+2} \mathrm{~A}_{1}$ and $\tilde{\mathrm{A}}^{+}{ }^{2} \mathrm{~B}_{2}$ states are shown as calculated using the CASPT2 method in $C_{2 \mathrm{v}}$ symmetry at an O-O bond length of $r=1.27$ $\AA$. The minima of the potential curves were calculated at $(r, \alpha)=\left(1.250 \AA, 132.11^{\circ}\right)$ and $\left(1.288 \AA, 104.05^{\circ}\right)$ for the $\tilde{\mathrm{X}}^{+}$and $\tilde{\mathrm{A}}^{+}$states, respectively. Because of their different symmetries in $C_{2 \mathrm{v}}(\mathrm{M})$, the $\tilde{\mathrm{X}}^{+}$and $\tilde{\mathrm{A}}^{+}$states can cross and exhibit a conical intersection at $\alpha=115.0^{\circ}$ located $2600 \mathrm{~cm}^{-1}$ above the $\tilde{\mathrm{X}}^{+}$state minimum at a bond length of $r=1.27 \AA$. The crossing occurs at a seam along the symmetric stretching coordinate [20]. Because the $\tilde{\mathrm{X}}^{+2} \mathrm{~A}_{1}$ and $\tilde{\mathrm{A}}^{+2} \mathrm{~B}_{2}$ states correlate with the $1 \mathrm{~A}^{\prime}$ and $2 \mathrm{~A}^{\prime}$ states in $C_{\mathrm{s}}$ symmetry $\left(r_{1} \neq r_{2}\right)$, they can interact and the crossing of the potentials is avoided as soon as the geometry deviates from the $C_{2 \mathrm{v}}$ symmetry.

To distinguish the adiabatic from the diabatic states (which, e.g., correspond to the 
bending potential curves displayed in Fig. 8), the lowest two adiabatic states are labeled as $1 \mathrm{~A}^{\prime}$ and $2 \mathrm{~A}^{\prime}$. In the adiabatic picture, the potential wells corresponding to the minima of the $\tilde{\mathrm{X}}^{+}$and $\tilde{\mathrm{A}}^{+}$states are located on the lowest surface and the seam of conical intersections forms a minimum energy path on the $2 \mathrm{~A}^{\prime}$ surface.

In Fig. 9, two-dimensional cuts through the adiabatic potential energy surface of the $1 \mathrm{~A}^{\prime}$ state along the $r_{1}$ and $\mathrm{r}_{2}$ coordinates are shown for fixed values of $\alpha$. These cuts have been calculated using the CASPT2 method. The potential is symmetric with respect to an interchange of $r_{1}$ and $r_{2}$. In Fig. 9 (a), the bond angle was kept fixed at a value of $\alpha=132.11^{\circ}$ corresponding to the equilibrium value of the $\tilde{\mathrm{X}}^{+}$state; the minimum on the potential energy surface can be seen at $r=r_{1}=r_{2}=1.240 \AA$ and exhibits $C_{2 \mathrm{v}}$ symmetry. The potential energy curve along the path which corresponds to the asymmetric stretching coordinate at small displacements from the equilibrium geometry is very flat and leads, via a low-lying saddle point, to a second minimum at large internuclear distances. This path corresponds to the dissociation coordinate of the reaction $\mathrm{O}_{3}^{+} \rightarrow \mathrm{O}_{2}^{+}+\mathrm{O}$. The minimum of the potential well corresponding to the $\tilde{\mathrm{X}}^{+}$state is only located about $1500 \mathrm{~cm}^{-1}$ below the saddle point which thus lies beneath the first dissociation threshold. The saddle point calculated at the MRCI/aug-cc-pVTZ level of theory is $\left(r_{1}, r_{2}, \alpha\right)=(1.354 \AA, 1.198$ $\AA, 124.282^{\circ}$ ) and is located $1534 \mathrm{~cm}^{-1}$ above the minimum of the $\tilde{\mathrm{X}}^{+}$state.

A cut at the equilibrium bond angle of the $\tilde{\mathrm{A}}^{+}$state $\left(\alpha=104.05^{\circ}\right)$ is shown in Fig. 9 (b). The minimum of the $\tilde{\mathrm{A}}^{+}$state is located at $r=r_{1}=r_{2}=1.288 \AA$ and also exhibits $C_{2 \mathrm{v}}$ symmetry. This potential well is very flat and the minimum energy path runs via a low lying saddle point along the dissociation coordinate. The discrepancy between the 
experimental $r_{0}\left(1.37(5) \AA, 111.3(38)^{\circ}\right)$ and the calculated $r_{\text {e }}$ geometries $\left(1.283 \AA, 104.14^{\circ}\right)$ of this state can be attributed to the marked anharmonicity of the potential in the vicinity of the equilibrium geometry. Only one bound state could be observed experimentally in this potential well, i.e., $\tilde{\mathrm{A}}^{+}(0,0,0)$.

The region around the saddle point close to the minimum of the $\tilde{\mathrm{X}}^{+}$state is displayed in Fig. 10. This cut has been calculated at the CASSCF $(11,9)$ level of theory. The second bond length $r_{2}$ was kept fixed at $r_{2}=1.19 \AA$ and $r_{1}$ and $\alpha$ were varied. The potential well of the $\tilde{\mathrm{X}}^{+}$state can be discerned around $\alpha=132^{\circ}$. The minimum energy path leads over the saddle point to a second minimum at large internuclear distances. The minimum corresponding to the $\tilde{\mathrm{A}}^{+}$state is not observable on this cut, but the path that leads to this minimum is discernible in the lower left corner of the figure.

The topological features of the lowest adiabatic potential energy surface provide a qualitative explanation for the sudden reduction of the rotational constants of the bands at excitation energies of more than $1500 \mathrm{~cm}^{-1}$ above the cationic ground state: The nuclear motion in this region is likely to spread over several wells on the potential surface and to reach into the region of large internuclear distances. We believe that this delocalization is responsible for the congested rotational structure observed in the PFI-ZEKE photoelectron spectra. First indications that the potential energy surface of the $1 \mathrm{~A}^{\prime}$ state is very flat were already given by Schmelz et al. [20] but have not been studied in detail.

In the wavenumber region close to the conical intersection (about $2600 \mathrm{~cm}^{-1}$ above the minimum of the $\tilde{\mathrm{X}}^{+}$state), non-adiabatic effects are expected to strongly influence the vibronic structure, and the nuclear motion is no longer confined to a single potential 
energy surface [49, 27]. The high density of vibronic states observed at these excitation energies, the irregular spacings between the bands and the apparently perturbed rotational structures of the $\tilde{\mathrm{X}}^{+}(0,3,0)$ and $(0,4,0)$ bands can thus be explained qualitatively by the complex topography of the potential energy surface and the occurrence of vibronic coupling.

The vibronic structure has already been modeled by Müller, Köppel and Cederbaum who also considered the non-adiabatic coupling between the $\tilde{\mathrm{X}}^{+}$and $\tilde{\mathrm{A}}^{+}$states $[25,26,27]$. The calculations were performed using an analytical potential energy surface that was adjusted to the $a b$ initio points calculated by Schmelz et al. [20] in $C_{2 \mathrm{v}}$ geometry. A harmonic potential function was assumed along the asymmetric stretching coordinate which does not include the low-lying barriers, so that the nuclear motion was confined to relatively small displacements in $r_{1}$ and $r_{2}$. The present results show that the nuclear motion covers much larger regions of the potential energy surface already at low excitation energies and that the long-range part of the potentials must also be considered in vibronic structure calculations.

Because of the complexity of the problem, an assignment of the higher excited vibronic bands will only be possible through model calculations of the vibronic structure which take into account the non-adiabatic coupling between the $\tilde{\mathrm{X}}^{+}$and $\tilde{\mathrm{A}}^{+}$states on global potential energy surfaces. Because of the high resolution achieved in the present study, it is desirable that these surfaces be calculated with spectroscopic accuracy $\left(\mathrm{a}\right.$ few $\left.\mathrm{cm}^{-1}\right)$ in order to make contact with the experimental data. Such calculations have recently become possible on molecules of comparable size as has been demonstrated in the case of $\mathrm{O}_{3}, \mathrm{NO}_{2}, \mathrm{H}_{2} \mathrm{O}$ and $\mathrm{NH}_{3}[24,9,50,51]$. 


\section{$5 \quad$ Summary and Conclusions}

The analysis of the rotationally resolved PFI-ZEKE photoelectron spectrum of $\mathrm{O}_{3}$ has established for the first time the symmetry assignment of the lowest two electronic states of the ozone cation. The $\tilde{\mathrm{X}}^{+}{ }^{2} \mathrm{~A}_{1}$ and $\tilde{\mathrm{A}}^{+}{ }^{2} \mathrm{~B}_{2}$ states are almost degenerate with an energy difference of only $T_{0} / h c=1089.7(4) \mathrm{cm}^{-1}$. The precise value of the adiabatic ionization energy determined here enabled the determination of a more accurate value for the lowest dissociation threshold of $\mathrm{O}_{3}^{+}\left(D_{0} / h c=4898(3) \mathrm{cm}^{-1}\right)$ using a thermochemical cycle. The ozone cation is thus only a very weakly bound molecule with a particularly flat and anharmonic ground state potential energy surface. The $a b$ initio calculations performed in the present study show that the potential energy surfaces of the $\tilde{\mathrm{X}}^{+}$and $\tilde{\mathrm{A}}^{+}$states cross each other about $2600 \mathrm{~cm}^{-1}$ above the minimum of the $\tilde{\mathrm{X}}^{+}$state. In $C_{\mathrm{s}}$ symmetry, both states transform under the same irreducible representation $\mathrm{A}^{\prime}$ and the crossing is avoided, which leads to low-lying saddle points on the adiabatic potential energy surface of the $1 \mathrm{~A}^{\prime}$ state. These barriers connect the potential wells of the $\tilde{\mathrm{X}}^{+}$and $\tilde{\mathrm{A}}^{+}$states with each other and with the region of large internuclear distances.

In the potential well corresponding to the $\tilde{\mathrm{X}}^{+}$state, a progression in the bending mode was observed up to $v_{2}^{+}=4$, whereas only one bound level was observed in the potential well corresponding to the $\tilde{\mathrm{A}}^{+}$state. The analysis of the rotational structures of the bands shows that the minima of both potential wells exhibit a $C_{2 \mathrm{v}}$ geometry and that the rovibrational structure is regular at excitation energies up to $\approx 1200 \mathrm{~cm}^{-1}$ above the cationic ground state. 
At excitation energies above $1500 \mathrm{~cm}^{-1}$, the nuclear motion starts spreading over a large region of the potential energy surface covering several minima also at large internuclear distances. The minima at large $r_{1}$ and $r_{2}$, which have been described for the first time in the present study, were not considered in earlier calculations of the vibronic structure of the $\tilde{\mathrm{X}}^{+}$ and $\tilde{\mathrm{A}}^{+}$states. We believe that the dense and complex pattern of observed spectral features in this region arises from this large amplitude motion and the non-adiabatic coupling in the vicinity of the crossing seam between the $\tilde{\mathrm{X}}^{+}$and $\tilde{\mathrm{A}}^{+}$states.

$\mathrm{O}_{3}^{+}$represents a highly interesting molecule which exhibits a very complex intramolecular dynamics already at low excitation energies: vibronic coupling between two electronic states in the vicinity of a conical intersection, large amplitude motions covering several minima on the potential energy surface and reaching into the region of large internuclear distances and a very low dissociation energy. The present study represents a first step toward the characterization of this complex molecular dynamics. Further theoretical studies would be desirable to aid in the understanding of the vibronic structure at higher excitation energies.

\section{Acknowledgements}

We thank René Gunzinger for his help in the development of the apparatus, and Prof. H. F. Schaefer III, Athens, and Dr. T. K. Ha, Zürich, for useful discussions concerning the $a b$ initio calculations. This work is supported financially by the Swiss National Science Foundation and the ETH Zurich. 


\section{References}

[1] R. P. Wayne, Chemistry of Atmospheres, Third Edition, Oxford University Press, Oxford, 2000 .

[2] R. Bacis, A. J. Bouvier, and J. M. Flaud, Spectrochim. Acta A 54, 17 (1998).

[3] J. M. Flaud and R. Bacis, Spectrochim. Acta A 54, 3 (1998).

[4] M.-R. De Backer-Barilly, A. Barbe, V. G. Tyuterev, and M.-T. Bourgeois, J. Mol. Spectrosc. 221, 174 (2003).

[5] M. H. Palmer and A. D. Nelson, Mol. Phys. 100, 3601 (2002).

[6] G. de Petris, Mass Spectrom. Rev. 22, 251 (2003).

[7] D. K. Hsu, D. L. Monts, and R. N. Zare, editors, Spectral Atlas of $\mathrm{NO}_{2} 5530$ to 6480 A, Academic Press, New York, 1978.

[8] B. Kirmse, A. Delon, and R. Jost, J. Chem. Phys. 108, 6638 (1998).

[9] V. Kurkal, P. Fleurat-Lessard, and R. Schinke, J. Chem. Phys. 119, 1489 (2003).

[10] T. N. Radwan and D. W. Turner, J. Chem. Soc. A, 85 (1966).

[11] C. R. Brundle, Chem. Phys. Lett. 26, 25 (1974).

[12] J. M. Dyke, L. Golob, N. Jonathan, A. Morris, and M. Okuda, J. Chem. Soc. Faraday Trans. 70, 1828 (1974). 
[13] D. C. Frost, S. T. Lee, and C. A. McDowell, Chem. Phys. Lett. 24, 149 (1974).

[14] S. Katsumata, H. Shiromaru, and T. Kimura, Bull. Chem. Soc. Jpn. 57, 1784 (1984).

[15] T. Cvitaš, L. Klasinc, and B. Kovač, Int. J. Quant. Chem. 29, 657 (1986).

[16] M. J. Weiss, J. Berkowitz, and E. H. Appelman, J. Chem. Phys. 66, 2049 (1977).

[17] A. Mocellin, K. Wiesner, F. Burmeister, O. Björneholm, and A. N. de Brito, J. Chem. Phys. 115, 5041 (2001).

[18] J. T. Moseley, J.-B. Ozenne, and P. C. Cosby, J. Chem. Phys. 74, 337 (1981).

[19] M. Probst, K. Hermansson, J. Urban, P. Mach, D. Muigg, G. Denifl, T. Fiegele, N. J. Mason, A. Stamatovic, and T. D. Märk, J. Chem. Phys. 116, 984 (2002).

[20] T. Schmelz, G. Chambaud, P. Rosmus, H. Köppel, L. Cederbaum, and H.-J. Werner, Chem. Phys. Lett. 183, 209 (1991).

[21] N. Kosugi, H. Kuroda, and S. Iwata, Chem. Phys. 58, 267 (1981).

[22] P. Decleva, D. De Alti, and A. Lisini, J. Chem. Phys. 89, 367 (1988).

[23] P. J. Hay, T. H. Dunning, and W. A. Goddard, J. Chem. Phys. 62, 3912 (1975).

[24] R. Siebert, P. Fleurat-Lessard, R. Schinke, M. Bittererová, and S. C. Farantos, J. Chem. Phys. 116, 9749 (2002).

[25] H. Müller, H. Köppel, L. S. Cederbaum, T. Schmelz, G. Chambaud, and P. Rosmus, Chem. Phys. Lett. 197, 599 (1992). 
[26] H. Müller, H. Köppel, and L. S. Cederbaum, New. J. Chem. 17, 7 (1993).

[27] H. Müller, H. Köppel, and L. S. Cederbaum, J. Chem. Phys. 101, 10263 (1994).

[28] F. Merkt, A. Osterwalder, R. Seiler, R. Signorell, H. Palm, H. Schmutz, and R. Gunzinger, J. Phys. B: At. Mol. Opt. Phys. 31, 1705 (1998).

[29] G. Reiser, W. Habenicht, K. Müller-Dethlefs, and E. W. Schlag, Chem. Phys. Lett. 152, 119 (1988).

[30] U. Hollenstein, R. Seiler, H. Schmutz, M. Andrist, and F. Merkt, J. Chem. Phys. 115, $5461(2001)$.

[31] L. Zhu and P. M. Johnson, J. Chem. Phys. 94, 5769 (1991).

[32] F. M. Phelps, M.I.T. Wavelength Tables, Vol. 2, Wavelengths by Element, MIT Press, Cambridge MA, 1982.

[33] F. Merkt, R. Signorell, H. Palm, A. Osterwalder, and M. Sommavilla, Mol. Phys. 95, 1045 (1998).

[34] Y. Song, M. Evans, C. Y. Ng, C.-W. Hsu, and G. K. Jarvis, J. Chem. Phys. 111, 1905 (1999).

[35] R. Signorell and F. Merkt, Faraday Discuss. 115, 205 (2000).

[36] R. Signorell and F. Merkt, Mol. Phys. 92, 793 (1997).

[37] S. Willitsch, U. Hollenstein, and F. Merkt, J. Chem. Phys. 120, 1761 (2004). 
[38] A. D. Buckingham, B. J. Orr, and J. M. Sichel, Phil. Trans. R. Soc. London A 268, $147(1970)$

[39] N. Taniguchi, K. Takahashi, Y. Matsumi, S. M. Dylewski, J. D. Geiser, and P. L. Houston, J. Chem. Phys. 111, 6350 (1999).

[40] P. Borowski, K. Andersson, P.-A. Malmqvist, and B. O. Roos, J. Chem. Phys. 97, $5568(1992)$

[41] I. Ljubić and A. Sabljić, Chem. Phys. Lett. 385, 214 (2004).

[42] H.-J. Werner and P. J. Knowles, J. Chem. Phys. 82, 5053 (1985).

[43] P. J. Knowles and H.-J. Werner, Chem. Phys. Lett. 115, 259 (1985).

[44] T. H. Dunning, J. Chem. Phys. 90, 1007 (1989).

[45] H.-J. Werner and P. J. Knowles, J. Chem. Phys. 89, 5803 (1988).

[46] P. J. Knowles and H.-J. Werner, Chem. Phys. Lett. 145, 514 (1988).

[47] H.-J. Werner, Mol. Phys. 89, 645 (1996).

[48] R. D. Amos et al., MOLPRO, a package of ab initio programs designed by H.-J. Werner and P. J. Knowles, version 2002.1.

[49] H. Köppel, L. S. Cederbaum, and W. Domcke, Chem. Phys. Lett. 110, 469 (1984).

[50] H. Lin, W. Thiel, S. N. Yurchenko, M. Carvajal, and P. Jensen, J. Chem. Phys. 117, 11265 (2002). 
[51] O. L. Polyansky, A. G. Császár, S. V. Shirin, N. F. Zobov, P. Barletta, J. Tennyson, D. W. Schwenke, and P. J. Knowles, Science 299, 539 (2003). 


\section{Table captions}

Table 1: Rotational selection rules in terms of changes of the asymmetric top quantum numbers $\Delta K_{a}$ and $\Delta K_{c}$ for photoionization transitions from the $\tilde{\mathrm{X}}{ }^{1} \mathrm{~A}_{1}(0,0,0)$ vibronic ground state of neutral $\mathrm{O}_{3}$ to cationic levels with vibronic symmetries $\Gamma_{\mathrm{ve}}^{+}$. The selection rules have been derived in the molecular symmetry (MS) groups $C_{2 \mathrm{v}}(\mathrm{M})$ and $C_{\mathrm{s}}(\mathrm{M}) \cdot \ell$ denotes the orbital angular momentum quantum number of the photoelectron.

Table 2: Adiabatic ionization energies of ozone corresponding to the formation of the $\tilde{\mathrm{X}}^{+}{ }^{2} \mathrm{~A}_{1}$ and $\tilde{\mathrm{A}}^{+}{ }^{2} \mathrm{~B}_{2}$ states of $\mathrm{O}_{3}$ and rotational constants for the $\tilde{\mathrm{X}}^{+}{ }^{2} \mathrm{~A}_{1}(0,0,0)$ and $\tilde{\mathrm{A}}^{+}{ }^{2} \mathrm{~B}_{2}(0,0,0)$ states of $\mathrm{O}_{3}^{+}$.

Table 3: Rotational constants and positions relative to the ground vibronic state of the bending levels $\left(0, v_{2}^{+}, 0\right)$ of the $\tilde{\mathrm{X}}^{+2} \mathrm{~A}_{1}$ state of $\mathrm{O}_{3}^{+}$.

Table 4: Theoretical geometries, harmonic frequencies and energy difference between the $\tilde{\mathrm{X}}^{+}$and $\tilde{\mathrm{A}}^{+}$states $\Delta T_{\mathrm{e}}$ of $\mathrm{O}_{3}^{+}$calculated using different theoretical methods and the aug-cc-pVTZ basis set. 


\section{Figure Captions}

Figure 1: Schematic molecular orbital diagram of $\mathrm{O}_{3}$ and $\mathrm{O}_{3}^{+}$illustrating the pronounced configurational mixing of the low lying electronic states. The percent values indicated below each configuration corresponds the results of the CASSCF calculation on $\mathrm{O}_{3}[24]$ and $\mathrm{O}_{3}^{+}$(see Section 4).

Figure 2: Survey of the PFI-ZEKE photoelectron spectrum of jet-cooled $\mathrm{O}_{3}$ between 101000 and $104000 \mathrm{~cm}^{-1}$, covering the region from the first adiabatic ionization threshold to slightly beyond the predicted position of the conical intersection between the $\tilde{\mathrm{X}}^{+}{ }^{2} \mathrm{~A}_{1}$ and $\tilde{\mathrm{A}}^{+2} \mathrm{~B}_{2}$ potential energy surfaces. All observed spectral features except those explicitly assigned to $\mathrm{O}_{2}$ and $\mathrm{H}_{2} \mathrm{O}$ represent photoelectron bands of $\mathrm{O}_{3}$.

Figure 3: Comparison of the PFI-ZEKE photoelectron and MATI spectra of $\mathrm{O}_{3}$ demonstrating how the bands of $\mathrm{O}_{3}$ were distinguished from impurity bands.

Figure 4: Comparison of the PFI-ZEKE photoelectron spectrum (lower trace) and the photoionization spectrum (upper trace) of jet-cooled ozone showing the adiabatic onset of the $\tilde{\mathrm{X}}{ }^{1} \mathrm{~A}_{1} \rightarrow \tilde{\mathrm{X}}^{+}{ }^{2} \mathrm{~A}_{1}$ photoelectron transition. The arrow indicates the position of the 
onset of the HeI photoelectron spectrum of room-temperature $\mathrm{O}_{3}$.

Figure 5: High-resolution PFI-ZEKE photoelectron spectrum of the $\tilde{\mathrm{X}}{ }^{1} \mathrm{~A}_{1}(0,0,0) \rightarrow$ $\tilde{\mathrm{X}}^{+2} \mathrm{~A}_{1}(0,0,0)$ band of $\mathrm{O}_{3}$ showing the almost completely resolved rotational structure (top trace). The transitions are labeled using the notation $N_{K_{a}^{\prime \prime} K_{c}^{\prime \prime}}^{\prime \prime} \rightarrow N_{K_{a}^{+} K_{c}^{+}}^{+}$. The lower trace represents a simulation of the spectrum based on a quantitative model for the rotational line intensities in the photoelectron spectra of asymmetric top molecules.

Figure 6: High resolution PFI-ZEKE photoelectron spectrum of the $\tilde{\mathrm{X}}{ }^{1} \mathrm{~A}_{1}(0,0,0) \rightarrow$ $\tilde{\mathrm{A}}^{+}{ }^{2} \mathrm{~B}_{2}(0,0,0)$ band of $\mathrm{O}_{3}$ showing partially resolved rotational structure (upper trace). The transitions are labeled using the notation $N_{K_{a}^{\prime \prime} K_{c}^{\prime \prime}}^{\prime \prime} \rightarrow N_{K_{a}^{+} K_{c}^{+}}^{+}$. The lower trace represents a simulation of the spectrum based on a quantitative model for the rotational line intensities in the photoelectron spectra of asymmetric top molecules.

Figure 7: High-resolution PFI-ZEKE photoelectron spectrum of $\mathrm{O}_{3}$ in the region 102630-102680 $\mathrm{cm}^{-1}$ showing the partially resolved rotational structure typical of most bands located beyond $102500 \mathrm{~cm}^{-1}$.

Figure 8: Potential curves of the $\tilde{\mathrm{X}}^{+2} \mathrm{~A}_{1}$ and $\tilde{\mathrm{A}}^{+}{ }^{2} \mathrm{~B}_{2}$ states of $\mathrm{O}_{3}$ along the bending coordinate. The curves were calculated for $C_{2 \mathrm{v}}$ geometries at an $\mathrm{O}-\mathrm{O}$ bond length of $1.27 \AA$ 
using the CASPT2 method and the cc-pVQZ basis set.

Figure 9: Cuts through the adiabatic potential energy surface of the $1 \mathrm{~A}^{\prime}$ state of $\mathrm{O}_{3}^{+}$ at a bond angle of a) $\alpha=132.11^{\circ}$ and b) $\alpha=104.05^{\circ}$ calculated at the CASPT2 $/$ cc-pVQZ level of theory. The contour values are given in $\mathrm{cm}^{-1}$ and are referenced to the minimum of the $\tilde{\mathrm{X}}^{+}$state.

Figure 10: Cut through the adiabatic potential energy surface of the $1 \mathrm{~A}^{\prime}$ state of $\mathrm{O}_{3}^{+}$ at $r_{2}=1.20 \AA$ calculated at the CASSCF/cc-pVQZ level of theory. The contour values are given in $\mathrm{cm}^{-1}$ and are referenced to the minimum of the $\tilde{\mathrm{X}}^{+}$state. 
Table 1: Willitsch et al.

\begin{tabular}{c|c|cc|cc|c}
\hline & & \multicolumn{2}{|c|}{$\ell$ even } & \multicolumn{2}{|c|}{$\ell$ odd } & \\
MS group & $\Gamma_{\mathrm{ve}}^{+}$ & $\Delta K_{a}$ & $\Delta K_{c}$ & $\Delta K_{a}$ & $\Delta K_{c}$ & \\
\hline \hline$C_{2 \mathrm{v}}(\mathrm{M})$ & $\mathrm{A}_{1}$ & odd & odd & even & even & $\nu_{1}, \nu_{2}$ \\
& $\mathrm{~B}_{2}$ & even & odd & odd & even & $\nu_{3}$ \\
\hline$C_{\mathrm{s}}(\mathrm{M})$ & $\mathrm{A}^{\prime}$ & even, odd & odd & even, odd & even & $\nu_{1}, \nu_{2}, \nu_{3}$ \\
\hline
\end{tabular}


Table 2: Willitsch et al.

\begin{tabular}{c|c|c}
\hline & $\tilde{\mathrm{X}}^{+2} \mathrm{~A}_{1}$ & $\tilde{\mathrm{A}}^{+2} \mathrm{~B}_{2}$ \\
\hline \hline$I E^{a} / \mathrm{cm}^{-1}$ & $101020.5(5)$ & $102110.1(6)$ \\
$A^{+} / \mathrm{cm}^{-1}$ & $5.99(10)$ & $2.65(26)$ \\
$\frac{1}{2}\left(B^{+}+C^{+}\right) / \mathrm{cm}^{-1}$ & $0.398(24)$ & $0.41(10)$ \\
\hline
\end{tabular}

${ }^{a}$ Adiabatic ionization energy corrected for the field-induced shift of the ionization thresholds $\Delta \tilde{\nu}=$ $-1.28(45) \mathrm{cm}^{-1}$. 
Table 3: Willitsch et al.

\begin{tabular}{c|cc|cc}
\hline Band & $\tilde{\nu}_{0}{ }^{a} / \mathrm{cm}^{-1}$ & $\Delta \tilde{\nu}_{0}\left(v_{2}^{+}, v_{2}^{+}-1\right)^{b} / \mathrm{cm}^{-1}$ & $A^{+} / \mathrm{cm}^{-1}$ & $\frac{1}{2}\left(B^{+}+C^{+}\right) / \mathrm{cm}^{-1}$ \\
\hline \hline$(0,0,0)$ & 0 & 0 & $5.99(10)$ & $0.398(24)$ \\
$(0,1,0)$ & $616.8(6)$ & $616.8(6)$ & $6.21(13)$ & $0.40(14)$ \\
$(0,2,0)$ & $1219.9(5)$ & $603.1(5)$ & $5.87(13)$ & $0.390(95)$ \\
$(0,3,0)$ & $1815.3(6)$ & $595.4(5)$ & $5.35(14)$ & $0.38(11)$ \\
$(0,4,0)$ & $2465.5(6)$ & $650.3(6)$ & $5.43(18)$ & $0.39(12)$ \\
\hline
\end{tabular}

${ }^{a}$ Wavenumber difference with respect to the $\tilde{\mathrm{X}}^{+2} \mathrm{~A}_{1}(0,0,0)$ band.

${ }^{b}$ Wavenumber difference $\tilde{\nu}_{0}\left(0, v_{2}^{+}, 0\right)-\tilde{\nu}_{0}\left(0, v_{2}^{+}-1,0\right)$. 
Table 4: Willitsch et al.

\begin{tabular}{cc|ccc|c|c}
\hline & & CASSCF(17,12) & CASPT2(17,12) & MRCI $^{a}$ & Ref. [20] & Expt. \\
\hline \hline$\tilde{\mathrm{X}}^{+2} \mathrm{~A}_{1}$ & $r / \AA$ & 1.249 & 1.251 & 1.238 & 1.256 & $1.250(20)^{b}$ \\
& $\alpha / \mathrm{deg}$ & 130.40 & 131.24 & 131.22 & 130.0 & $131.49(91)^{b}$ \\
& \multirow{4}{*}{$\tilde{\nu}_{1}^{c} / \mathrm{cm}^{-1}$} & 1016 & & & 1002 & \\
& $\tilde{\nu}_{2}^{c} / \mathrm{cm}^{-1}$ & 634 & & & 592 & $616.8(6)^{d}$ \\
& $\tilde{\nu}_{3}^{c} / \mathrm{cm}^{-1}$ & 838 & & & & \\
\hline & $r / \AA$ & 1.291 & 1.297 & 1.283 & 1.290 & $1.370(52)^{b}$ \\
& $\alpha / \mathrm{deg}$ & 104.45 & 103.99 & 104.14 & 104.9 & $111.3(38)^{b}$ \\
\hline$\tilde{\mathrm{A}}^{+2} \mathrm{~B}_{2}$ & 1013 & 976 & 1038 & & $1089.7(4)^{e}$ \\
\hline
\end{tabular}

${ }^{a}$ Only configuration state functions with coefficients $>0.01$ were retained in the calculation.

${ }^{b} r_{0}$ structures

${ }^{c}$ Harmonic wavenumber

${ }^{d}$ Fundamental wavenumber

${ }^{e} \Delta T_{0}$ 
Figure 1: Willitsch et al.

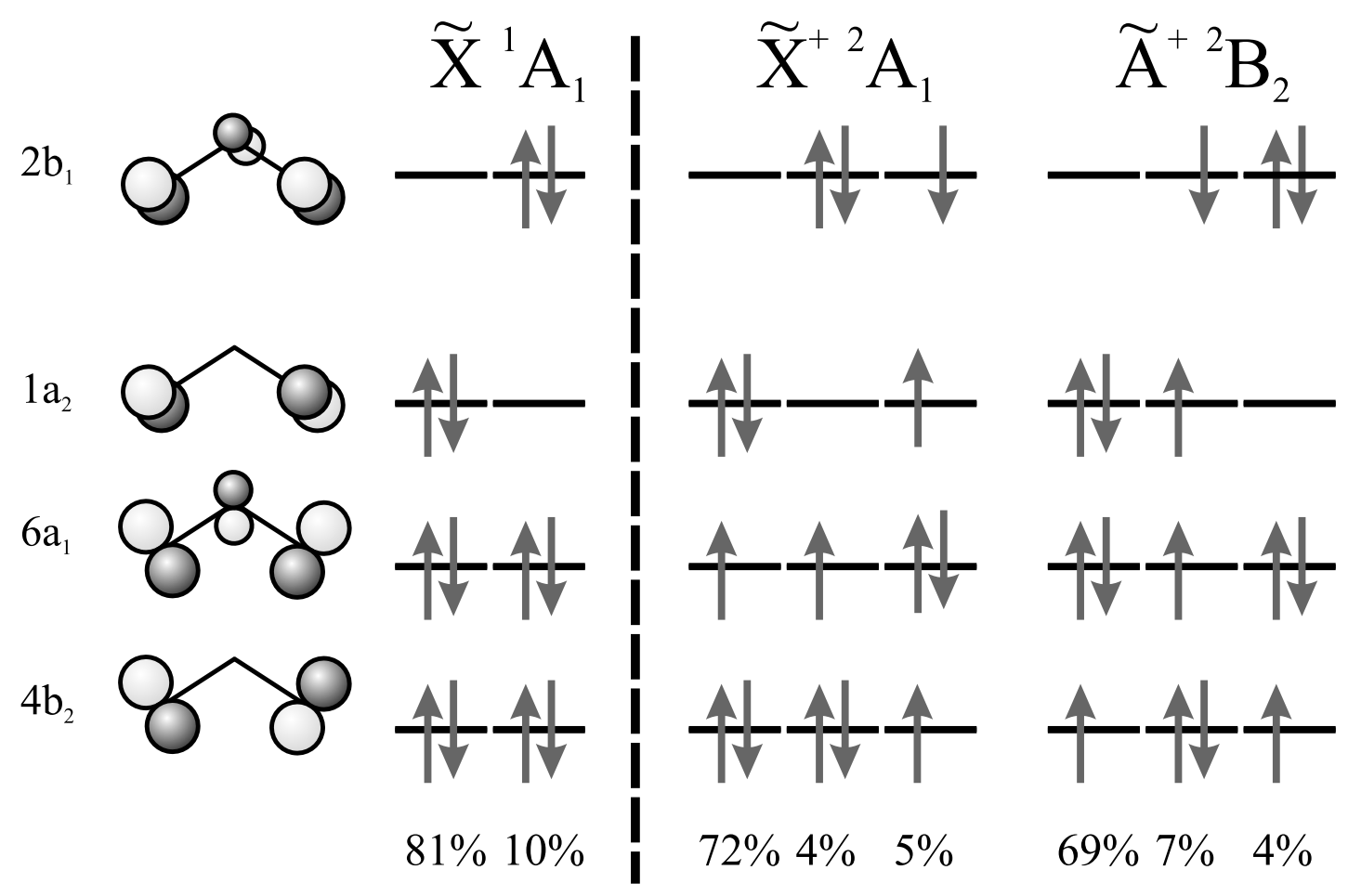


Figure 2: Willitsch et al.

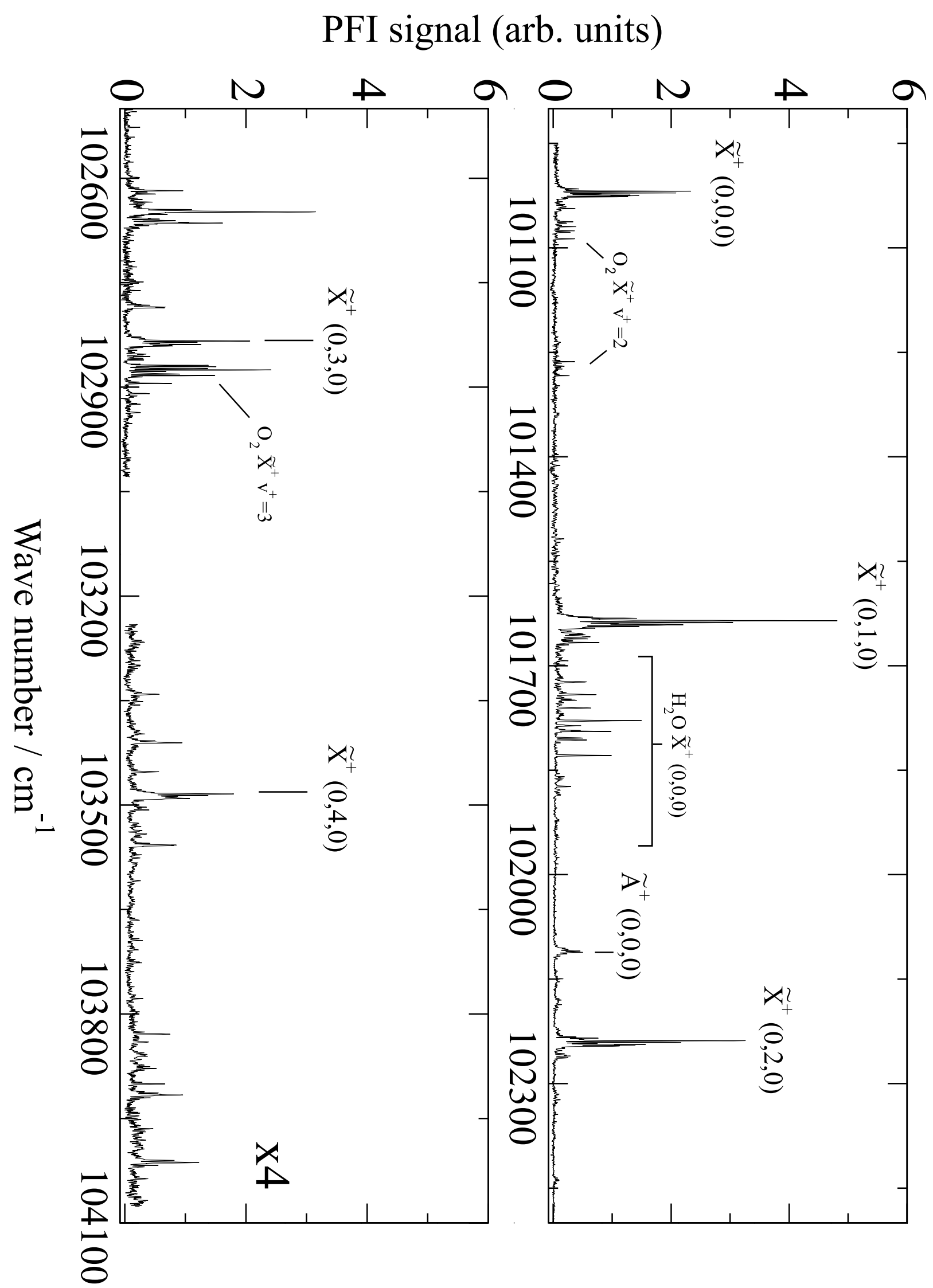


Figure 3: Willitsch et al.

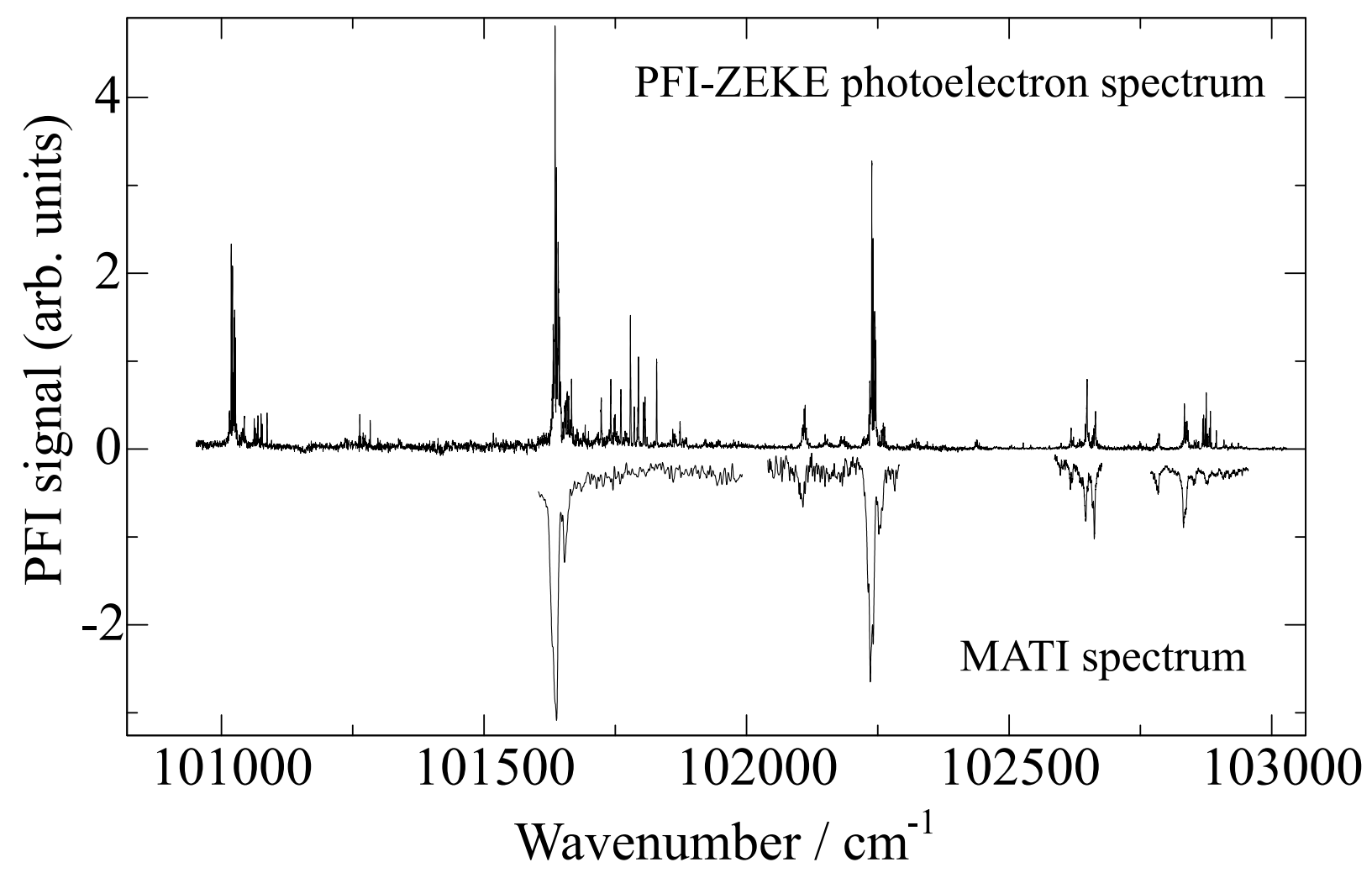


Figure 4: Willitsch et al.

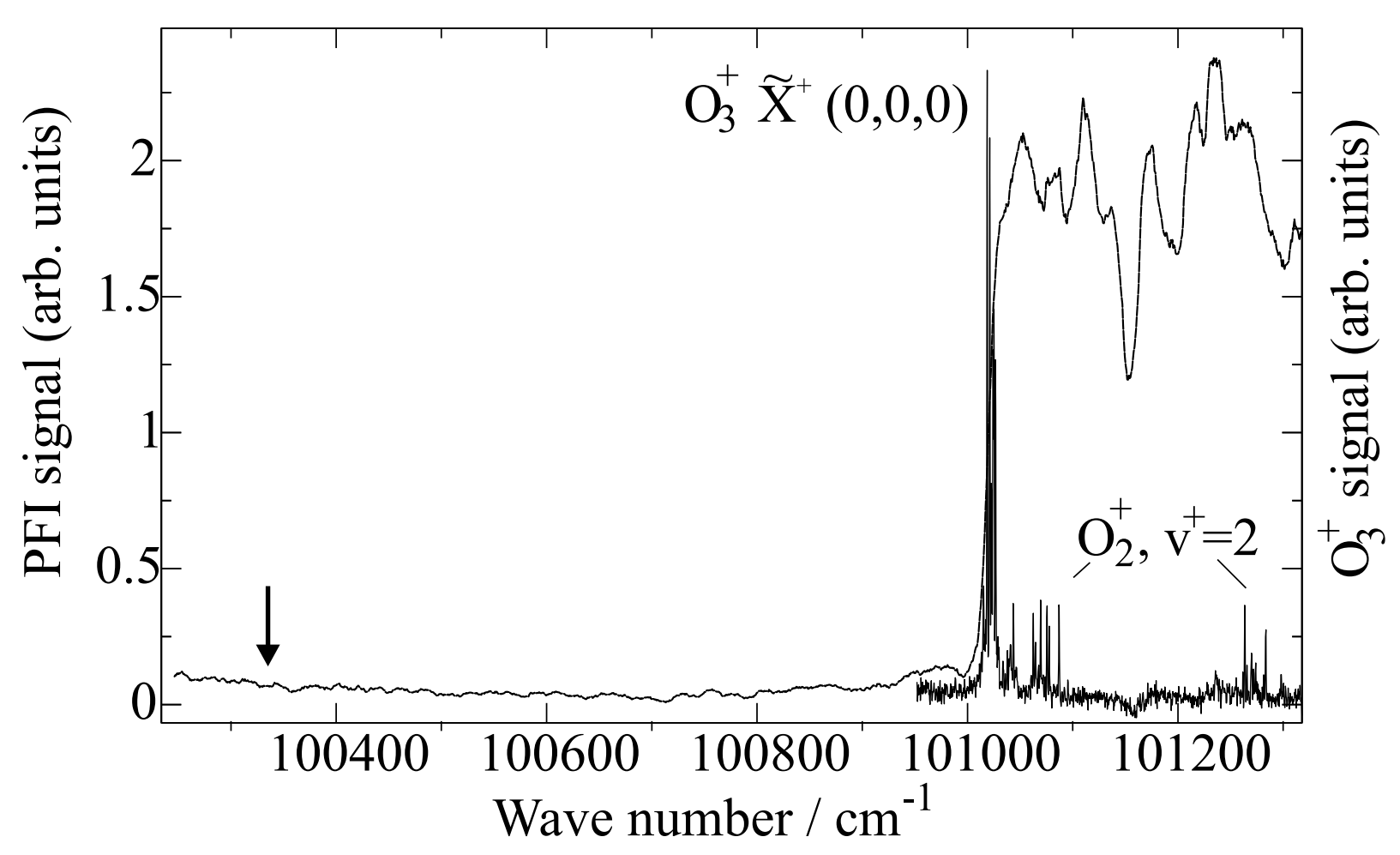


Figure 5: Willitsch et al.

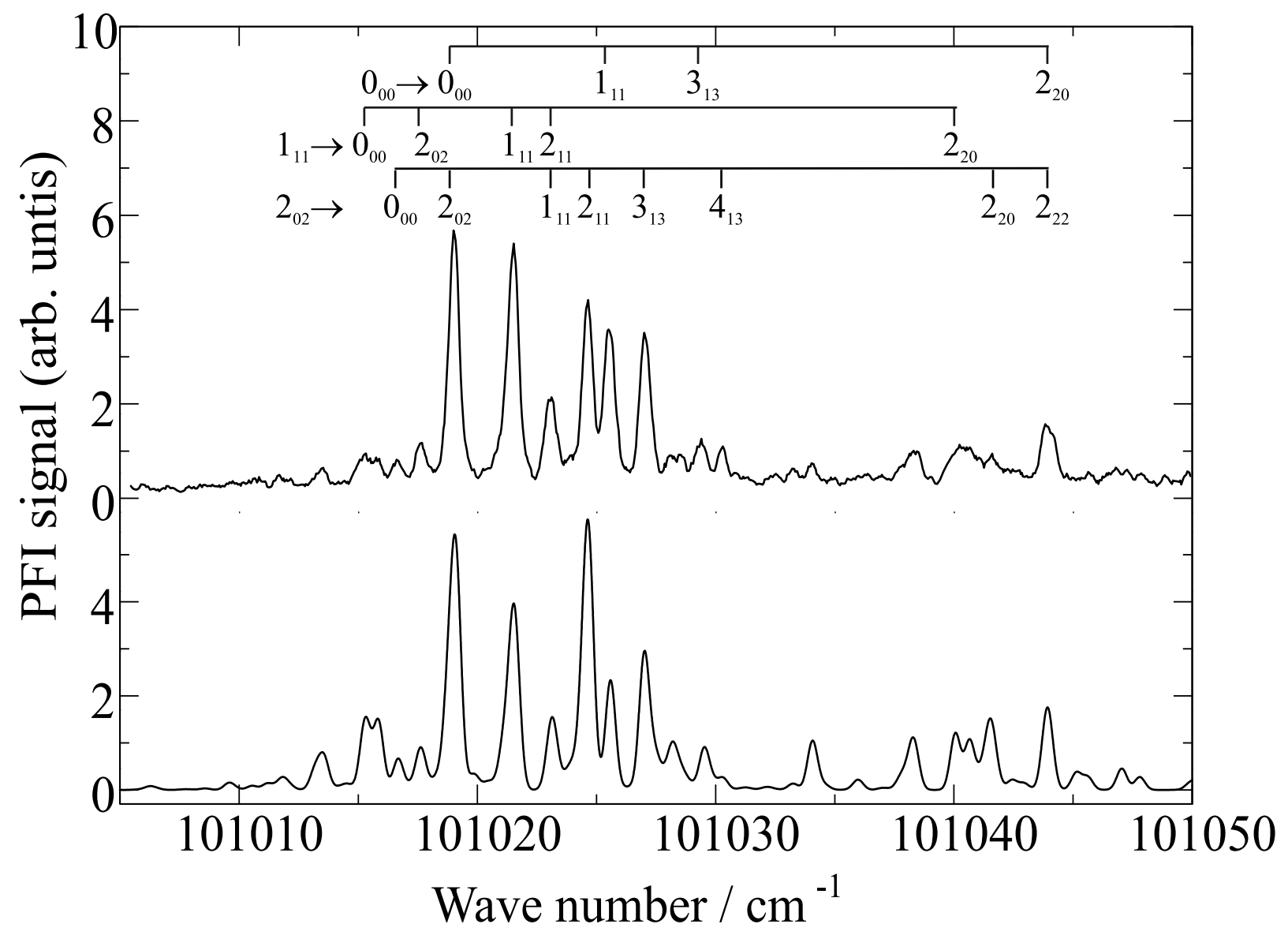


Figure 6: Willitsch et al.

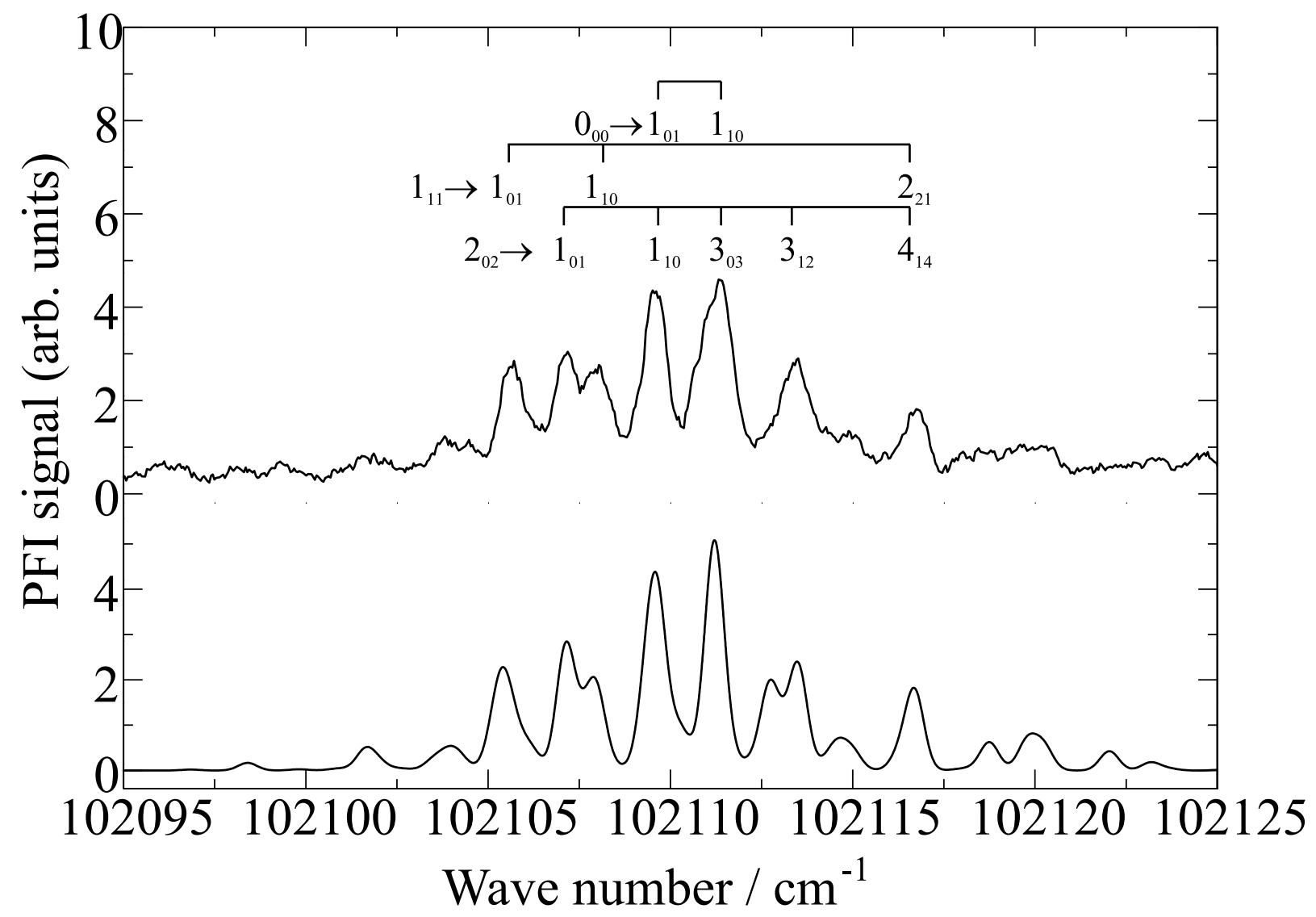


Figure 7: Willitsch et al.

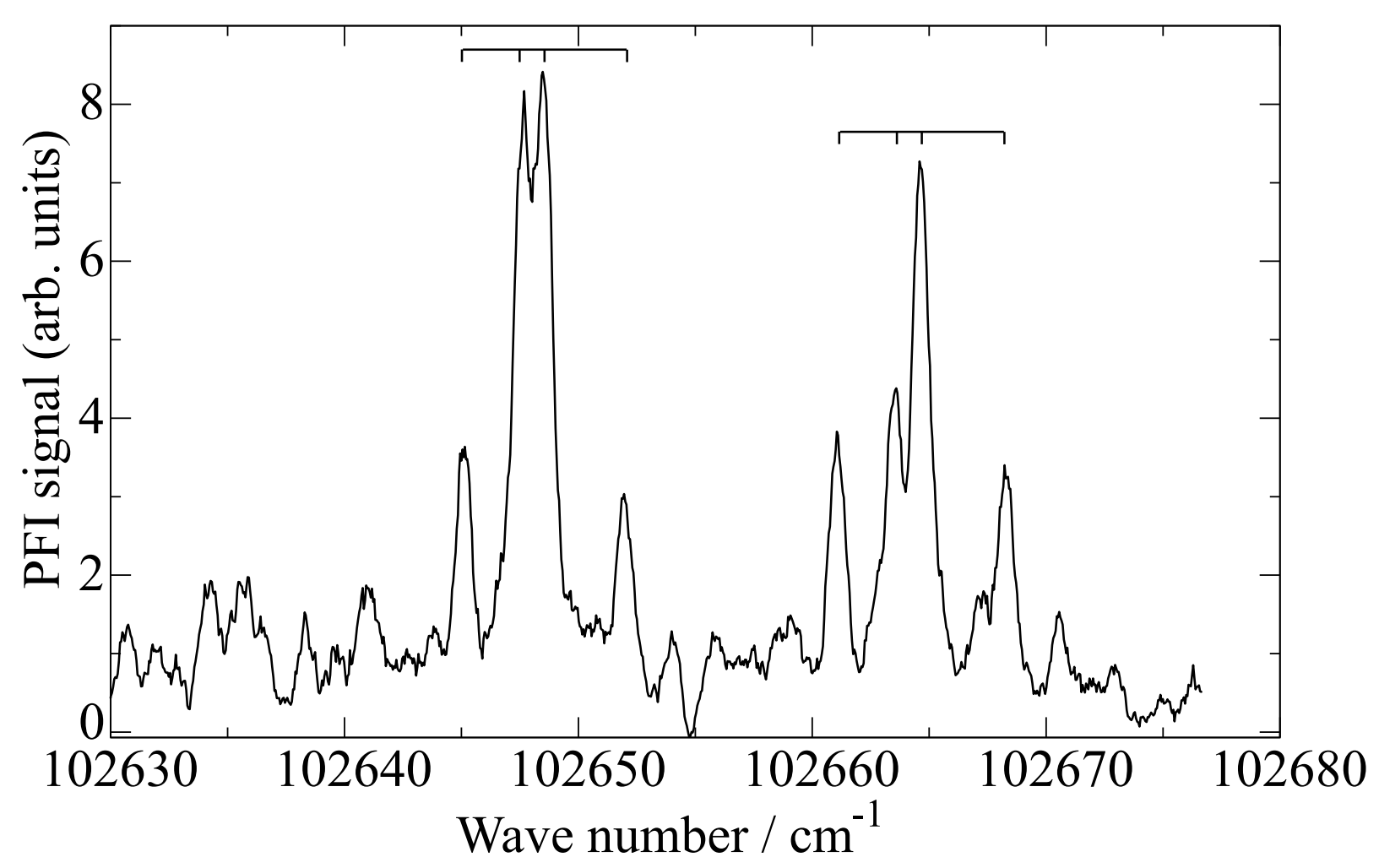


Figure 8: Willitsch et al.

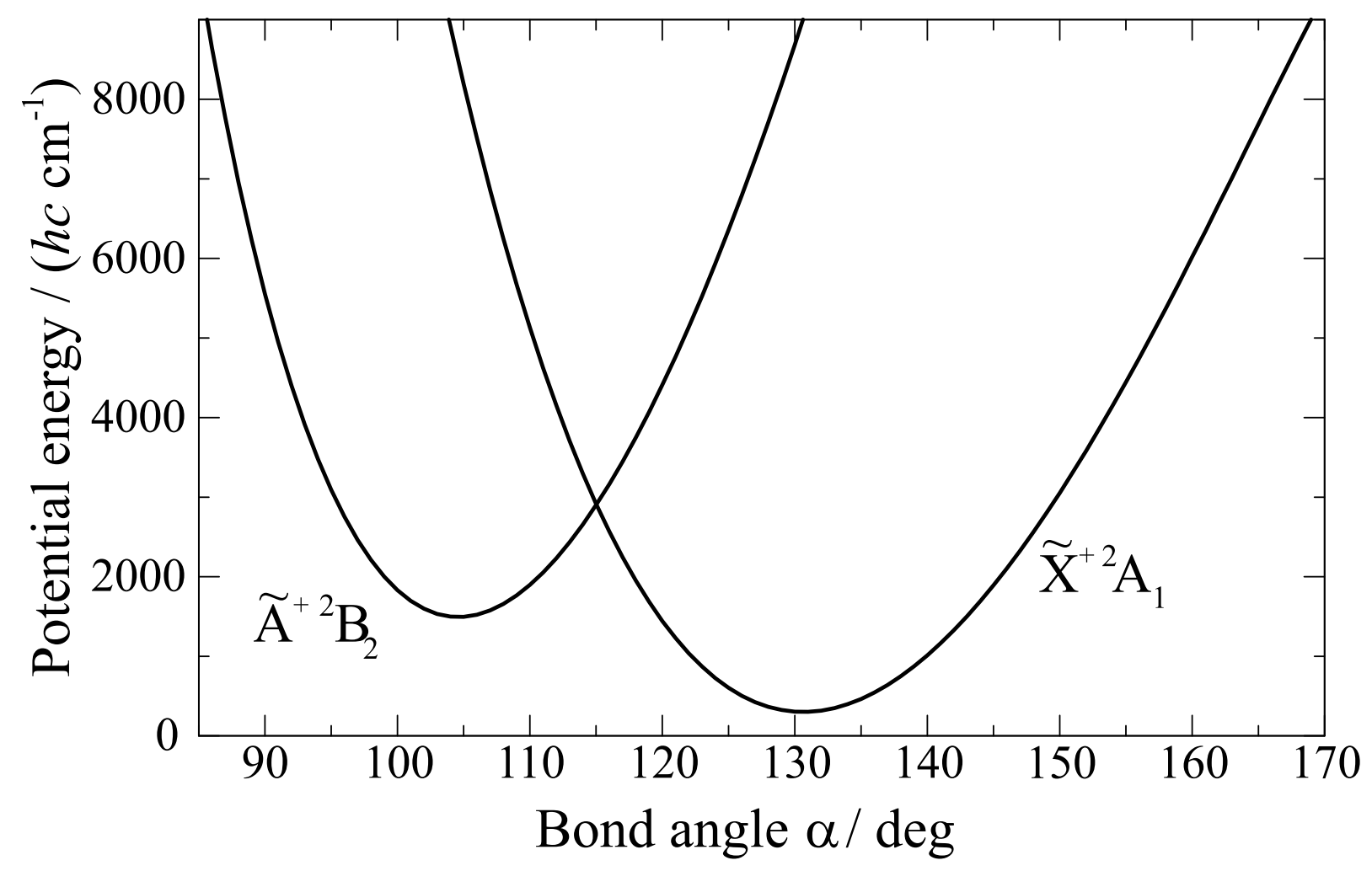


Figure 9: Willitsch et al.
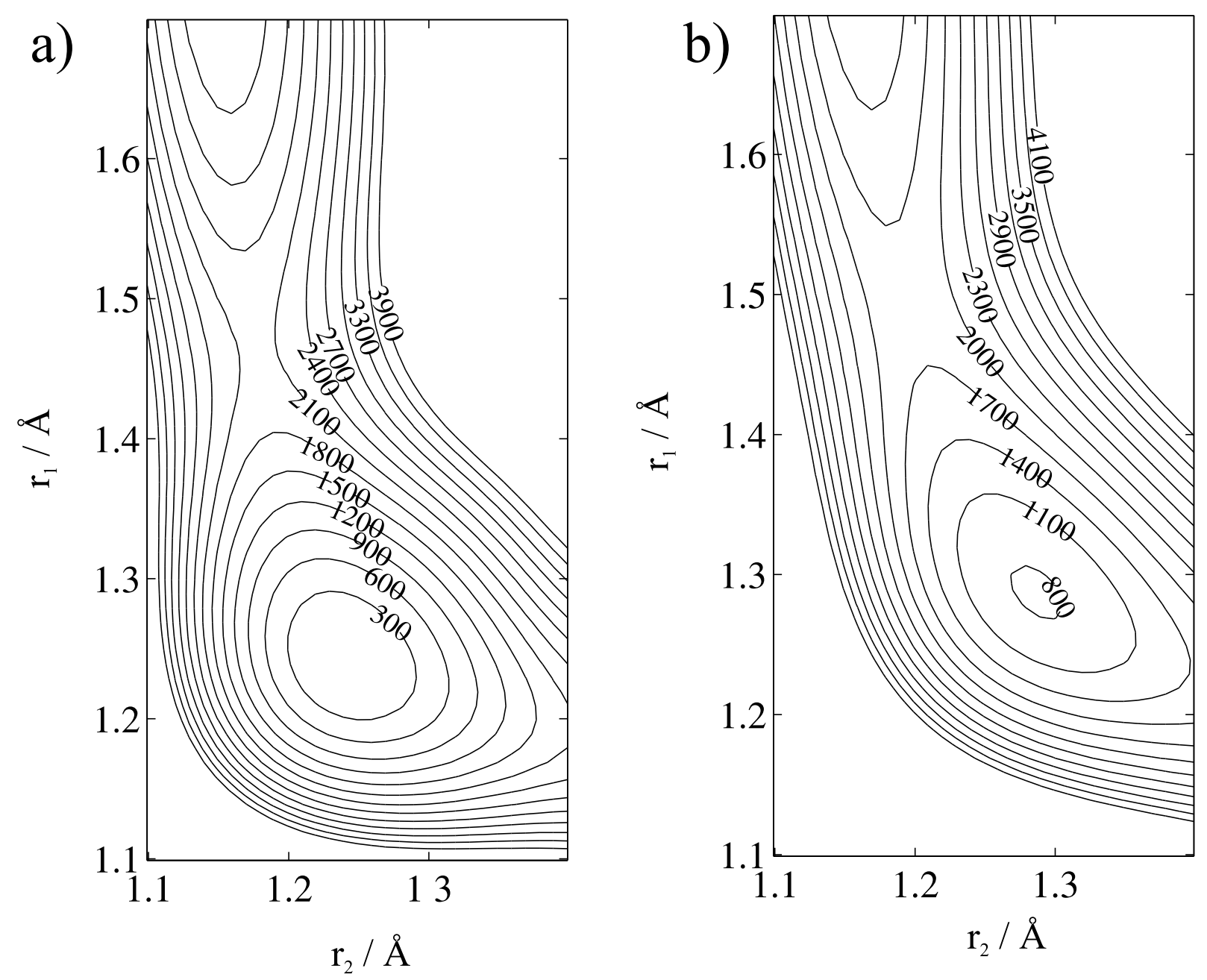
Figure 10: Willitsch et al.

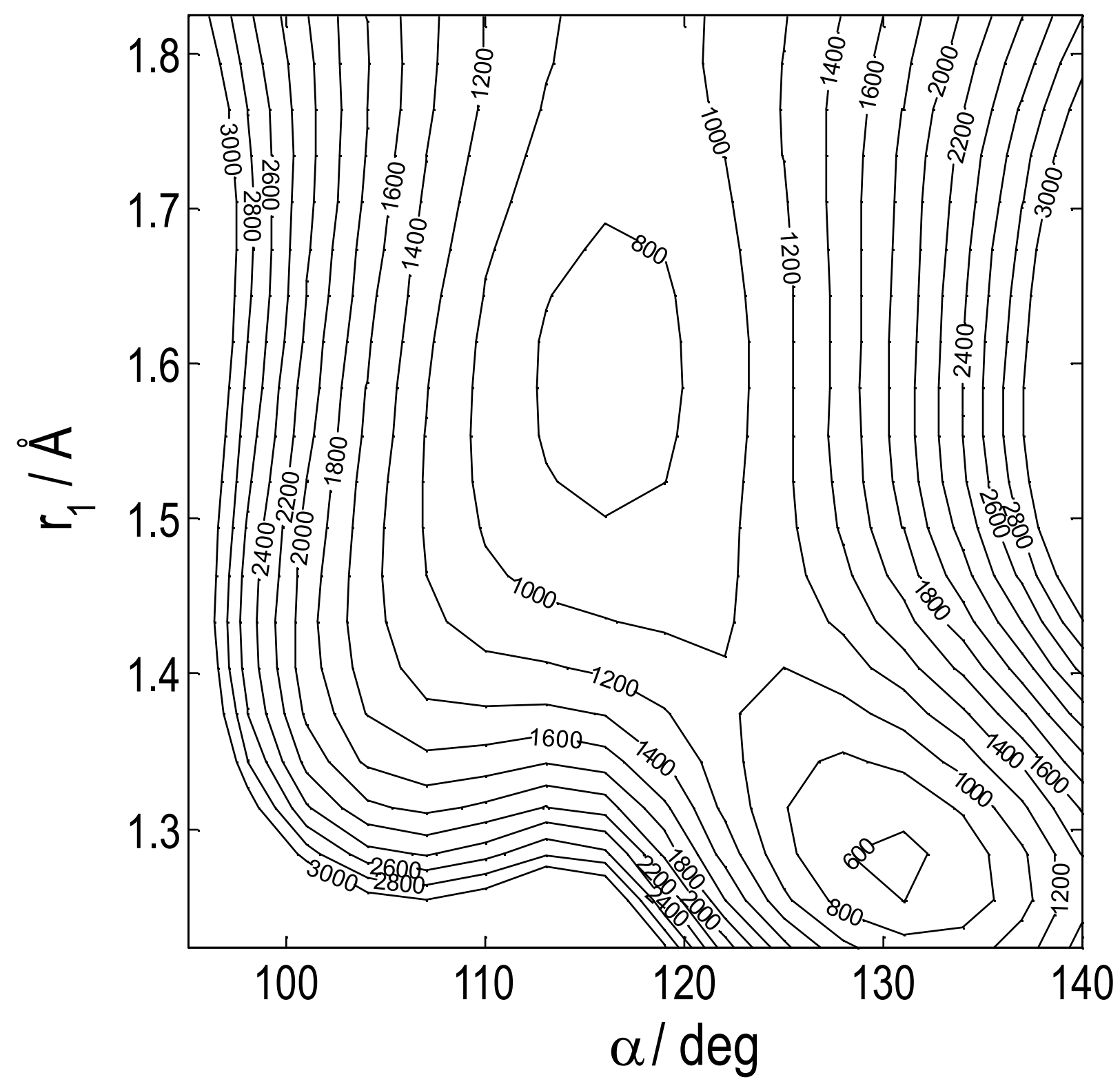

